\title{
Longitudinal Axis Display Requirements \\ for High speed Cruise
}

by

David Honaker

Thesis submitted to the Faculty of the

Virginia Polytechnic Institute and state University

in partial fulfillment of the requirements for the degree of Master of Science

in

Aerospace Engineering

APPROVED:

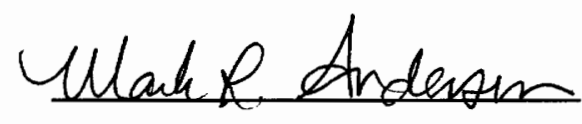

Dr. M. R. Anderson

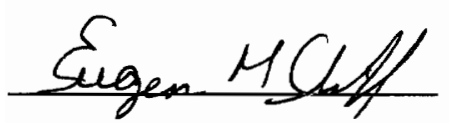

Dr. E. M. Cliff

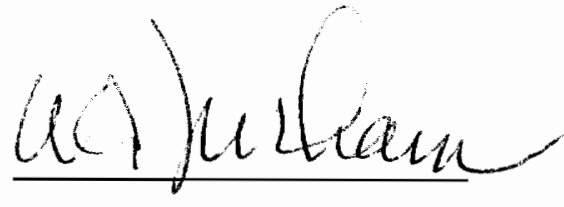

Dr. W. C. Durham

December 1993

Blacksburg, Virginia 
$c .2$

$\angle D$
5655
$V 855$
1993
$H 658$

C.2 


\section{Longitudinal Axis Display Requirements \\ for High speed Cruise}

by

David Honaker

Committee Chairman: Dr. M. R. Anderson Virginia Polytechnic Institute and State University

\section{(ABSTRACT)}

Altitude excursions particular to high speed aircraft are investigated in this thesis. An aerodynamic database of the $X B-70$ is created and a longitudinal linear model is constructed for a high speed cruise flight condition. An examination of the unpiloted aircraft dynamics revealed that the excursions were not due to a poorly handling aircraft. Thus, it is theorized that the excursions are due to pilotvehicle interactions. A classical control method developed a loop closure scheme suitable for acceptable control of the aircraft. The results showed that a pilot should close an inner loop with negative attitude feedback and an outer loop with positive flight path feedback. A modern control method analysis using an optimal control pilot model confirmed the preceding conclusions. Based on these results, the cockpit pitch attitude display resolution should be less than $1^{\circ}$ so that the pilot will be able to perform the loop closures necessary for constant altitude flight. 


\section{Acknowledgments}

I extend my thanks and gratitude to Dr. Mark $R$. Anderson for his invaluable insight, support and guidance throughout this research effort and my graduate studies. without him, this thesis would be impossible.

I would also like to thank Dr. Eugene M. Cliff and Dr. Wayne $\mathrm{c}$. Durham for being members of my graduate committee.

I wish to thank Mr. Warren Hutchinson for his friendship, instruction and guidance, for without whom I probably would not have attended college.

Thanks to Jonathan I. Blatt for his friendship and encouragement throughout the past $5 \frac{1}{2}$ years.

To my parents, Leslie c. Honaker and Bridget F. Honaker, I thank them for their love, support, and encouragement.

Most importantly, I thank God for giving me the talents, skills, and supporting loved ones that have all been necessary for this accomplishment. 


\section{Table of contents}

Item

Page

List of Tables vi

List of Figures

vii

List of symbols

$\mathbf{x}$

List of Abbreviations

xiv

1.0 Introduction

2.0 XB-70 Model Development

2.1 Aerodynamic Model

2.2 Pitch Axis Control system

3.0 Unpiloted Aircraft Dynamic Survey

4.0 Analysis with Sequential Pilot Loop Closure

4.1 Pitch Angle Loop Closure

4.1.1 Negative Pitch Angle Feedback

4.1.2 Positive Pitch Angle Feedback

4.2 Flight Path Angle Loop Closure

4.2.1 Negative Flight Path Feedback 
4.3 Sequential Loop Closure

4.3.1 Short Period Mode Results

4.3.2 Phugoid Mode Results

4.3.3 Sequential Loop Closure Discussion

5.0 Analysis with Modern Control Methods

5.1 Pilot Modeling

5.2 Task Modeling

5.3 Multiloop Pilot Analysis using MOCM

5.3.1 Methodology

6. 0 Conclusion

7.0 References

8.0 Appendix 1: State Space Models of the XB-70

9.0 Appendix 2: Turbulence Model 


\section{List of Tables}

Item

Page

Table 1: XB-70 Longitudinal Characteristics

Table 2: Short Period Mode Variation with Outer Loop Gain

Table 3: Phugoid Mode Variation with outer Loop Gain 32

Table 4: MOCM Parameters and constants 39

Table 5: Cruise Flight Condition 60 


\section{List of Figures}

Figure $\quad \underline{\text { Title }}$

Page

1-a $\quad \mathrm{XB}-70$ Supersonic Aircraft

4

1-b XB-70 Flight Envelope 4

2 Pitch Axis Control System 7

$\begin{array}{llll}3 & \text { Stability Augmentation System } & 8\end{array}$

4 Short-Term Pitch Response to Pitch

Controller Requirements 11

5 Short-Period Frequency Requirements

6-a Short Field Landing Technique 16

6-b Conventional Control Technique 16

7 Attitude Closure: Negative Feedback, Phugoid Mode Root Locus 20

8 Attitude Closure: Negative Feedback, Short Period Mode Root Locus 21

9 Attitude Closure: Positive Feedback, Phugoid Mode Root Locus

10 Attitude Closure: Positive Feedback, Short Period Mode Root Locus 
Flight Path Closure: Negative Feedback, Phugoid Mode Root Locus

Flight Path Closure: Negative Feedback, Short Period Mode Root Locus

Flight Path Closure: Positive Feedback, Phugoid Mode Root Locus

Flight Path Closure: Positive Feedback, Short Period Mode Root Locus

17 Pitch Attitude Command system

18 Comparison of Sample MOCM and OCM Results

22-a Flight Path rms versus Attitude Threshold 
25-a Flight Path Rate rms vs. Attitude Threshold 66

25-b Flight Path Rate rms vs. N

26-a Pitch Attitude rms vs. Attitude Threshold

26-b Pitch Attitude rms vs. N

27-a Pitch Rate rms vs. Attitude Threshold

68

27-b Pitch Rate rms vs. N

68

28-a Stick Force vs. Attitude Threshold

69

28-b Stick Force vs. N

69 


\section{List of symbols}

A Linear state matrix for $\mathrm{XB}-70$ with PACS

B Linear control input matrix for XB-70 with PACS

$\mathrm{E}_{\infty} \quad$ Steady state expected value

Fcc Pilot stick force (1b.)

$\mathrm{H}_{\gamma} \quad$ Total transfer function block from $\gamma$ to $\mathrm{F}_{\mathbf{C C}}$

$\mathrm{H}_{\theta}$ Total transfer function block from $\theta$ to $\mathbf{F}_{\mathbf{C C}}$

$I_{y}$ Moment of inertia about $y$-body axis (slug-ft ${ }^{2}$ )

$J$ Quadratic performance index or cost function

$\mathrm{K}_{\gamma} \quad$ Flight path feedback gain (dB)

$\mathrm{K}_{\theta} \quad$ Attitude feedback gain (dB)

$L_{W} \quad$ Turbulence length scale (ft)

M Mach number

$\mathrm{N} \quad$ Integer variable utilized in computations

Qy observation weighting matrix

s wing area $\left(f t^{2}\right)$

V Total trim velocity (ft/sec)

w Disturbance intensity

a $\mathrm{XB}-70$ linear state matrix,

$a_{Z} \quad$ Normal acceleration $\left(f t / \sec ^{2}\right)$, equal to $V(\dot{\alpha}-q)-1 \dot{q}$

$a_{Z}^{B} \quad$ Bobweight normal acceleration $\left(\mathrm{ft} / \mathrm{sec}^{2}\right), V(\dot{\alpha}-\mathrm{q})-1_{B} \dot{q}$

$a_{z}^{\prime} \quad$ Normal acceleration at $1^{\prime}\left(\mathrm{ft} / \mathrm{sec}^{2}\right), V(\dot{\alpha}-\mathrm{q})-1^{\prime} \dot{q}$

$a_{\mathrm{TH}}$ Perception threshold $\left({ }^{\circ}\right.$ or $\%$ sec)

b $\mathrm{XB}-70$ linear control input matrix

c Mean aerodynamic chord (ft)

f Control rate weight, attention allocation 
$f(s)$ Turbulence model filter

h Altitude ( $f t$ )

1 Moment arm for normal acceleration computation ( $f t$ )

$l_{B}$ Moment arm for bobweight normal acceleration ( $f t$ )

$I^{\prime}$ Moment arm for SAS normal accelerometer ( $\mathrm{ft}$ )

$\mathrm{n} / \alpha$ Load factor per angle of attack (g/rad), $\frac{\mathrm{V}}{\mathrm{g}} \frac{1}{\tau_{\theta_{2}}}$

p Pilot

q Total and perturbed pitch rate ( $\mathrm{rad} / \mathrm{sec}$ )

$r$ Control defection weight

s La Place variable

u Linear system control vector, control

v' Total perturbed velocity (ft/sec)

w Downward component of velocity ( $\mathrm{ft} / \mathrm{sec}$ )

x Linear state vector

Y Observations, Linear output vector

$(0 / i)$ Transfer function from input $i$ to output o $\infty \quad$ Infinity

Greek Symbols:

$\Delta$ Denotes change in a particular variable

$\Phi \quad$ Turbulence spectra

$\Omega \quad$ Normalized frequency, $\omega / \mathrm{V}(1 / \mathrm{ft})$

$\alpha \quad$ Perturbed angle of attack (rad)

$\alpha_{g} \quad$ Gust disturbance in angle of attack (rad)

$\delta \quad$ Control deflection

$\gamma \quad$ Kinematic flight path angle (rad), $\theta-\alpha$ 


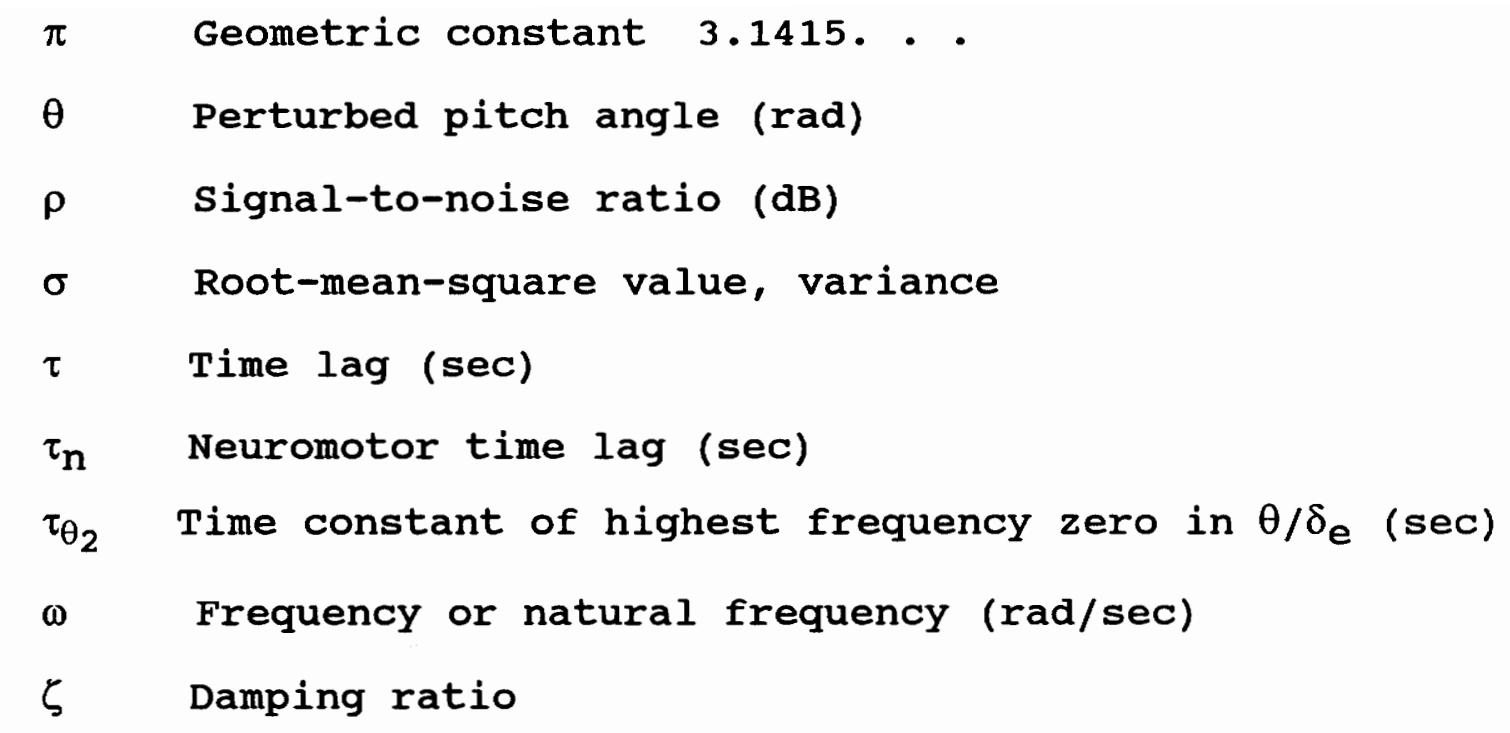

Subscripts:

B Bobweight

RMS RMS value with all thresholds set to zero

T wing -tip

TH Threshold value

c Canard

e Elevon, error signal

esAS Pitch SAS output

p Pilot

ph Phugoid mode

sp Short period mode

t Thrust

u Input, motor

w Downward component of perturbed velocity (ft/sec)

$w(t)$ White noise that drives disturbances

wg Gust in w ( $\mathrm{ft} / \mathrm{sec})$

Y output

$y_{i} i^{\text {th }}$ observation 
$\gamma \quad$ Flight path closure without attitude closure

$\gamma+\theta \quad$ Flight path closure with inner attitude closure Superscripts :

- Internal variables in PACS not due to Stick Force

* Internal variables in PACs due to stick Force

- Denotes particular accelerometer location

- Time rate of change

B Bobweight

T Transpose

$z \quad$ Denotes downward direction 


\section{List of Abbreviations}

HSCT High Speed civil Transport

LHP Left-Half-Plane

MOCM Modified Optimal Control Pilot Model

oCM Optimal Control Pilot Model

PACS Pitch Axis Control System

PIO Pilot Induced Oscillations

RHP Right-Half-Plane

SAS Stability Augmentation system

dB decibel

ft Feet

g Gravity, $32.2 \mathrm{ft} / \mathrm{sec}^{2}$

1b Pounds

rms Root-Mean-square

sec second

slug Mass unit for U.S. customary units (1b. $\sec ^{2} / f t$ ) 


\subsection{Introduction}

High speed aircraft such as the $X B-70$ and $S R-71$ have experienced problems with undesired altitude excursions during high speed cruise. The excursions were not due to the aircraft or the flight control system. Low resolution cockpit displays, inaccurate sensors, and sensor lags made accurate control of altitude at high speed cruise difficult for the pilots. As a result, the inadequate display characteristics resulted in pilot-induced oscillations (PIO) .

The problems associated with previous high speed aircraft gain renewed importance with the present joint government-industry research concerning the future High Speed Civil Transport (HSCT). The HSCT will be flown primarily by autopilot; however, manual control must be feasible throughout the flight envelope for safety reasons. At high altitudes there is virtually no perceptible horizon and the pilot will fly on instrumentation alone. Therefore, as evidenced by the $\mathrm{XB}-70$ and $\mathrm{SR}-71$ experiences, the instrumentation must be sufficiently accurate and reliable if the pilot is to achieve flight characteristics acceptable to civilian passengers. The HSCT's cockpit is expected to employ digital displays and television monitors. The HSCT engineers must consider how the monitors' refreshing rate and resolution will affect the pilot-aircraft interaction. 
An analysis of a proposed HSCT's performance will include aircraft handling qualities as well as the interaction of pilot and vehicle. Thus, a pilot model is required that will accurately predict a pilot's control strategy while taking into account the cockpit display characteristics. Therefore, the focus of this research is: Can the PIO behavior observed during high speed cruise of previous large aircraft be predicted using modern analysis methods? 


\subsection{XB-70 Kodel Development}

The XB-70 was chosen as a representative aircraft for this research because it is a supersonic aircraft that has experienced undesired performance during high speed flight. A literature review produced eighteen references that contain a wealth of XB-70 flight test data, wind tunnel data and predictions[1-18]. In addition, the references give insight to the problems encountered with the XB-70 flight test experience and some of the measures taken to alleviate those problems. Finally, the references provide a plethora of XB-70 aerodynamic properties.

Figure 1-a shows the three principle views of the XB70. As Figure 1-a shows, the $\mathrm{XB}-70$ has a long slender fuselage, a canard behind the pilot's station to provide pitch control, and the delta-wing configuration used widely for supersonic aircraft. In addition, six segmented elevons on the delta wing's trailing edge provide pitch and roll control while two rudders provide yaw control. The XB-70 is powered by six YJ93-GE-3 engines, each capable of nearly 30,000 1b. static thrust at sea level. Lastly, the $\mathrm{XB}-70$ wing-tip deflections reduce drag by capturing the engine nacelle shock and improve directional stability.

Figure $1-b$ shows the $x B-70$ 's flight envelope. The altitude versus Mach plot reveals the areas of the flight regime that the $\mathrm{xB}-70$ may travel in. As the figure shows, 


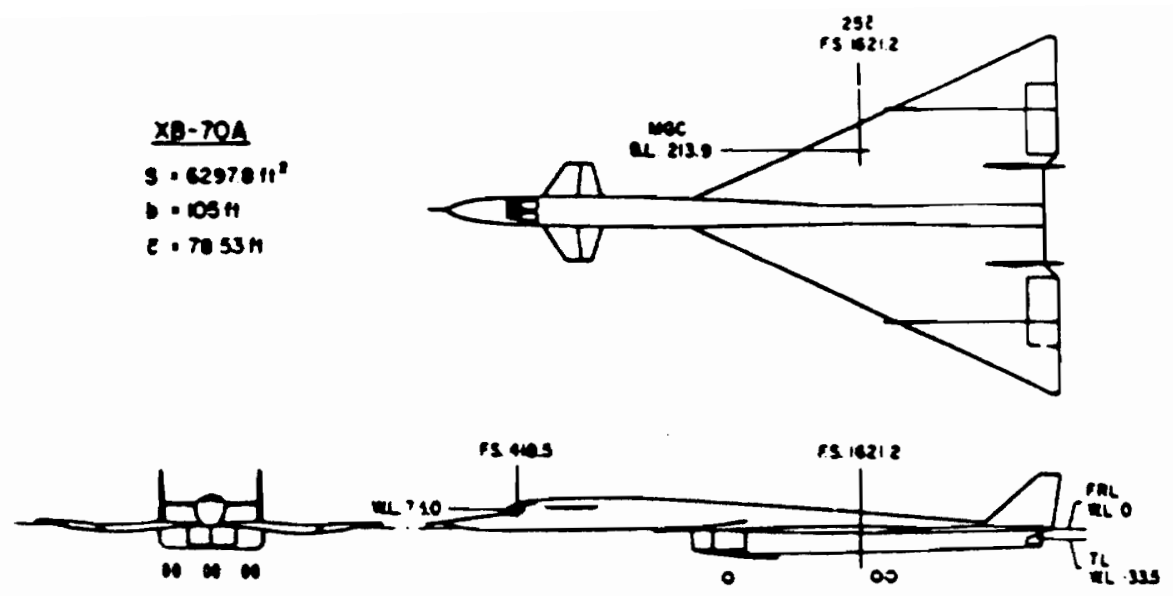

Figure 1-a: $\mathrm{XB}-70$ supersonic Aircraft

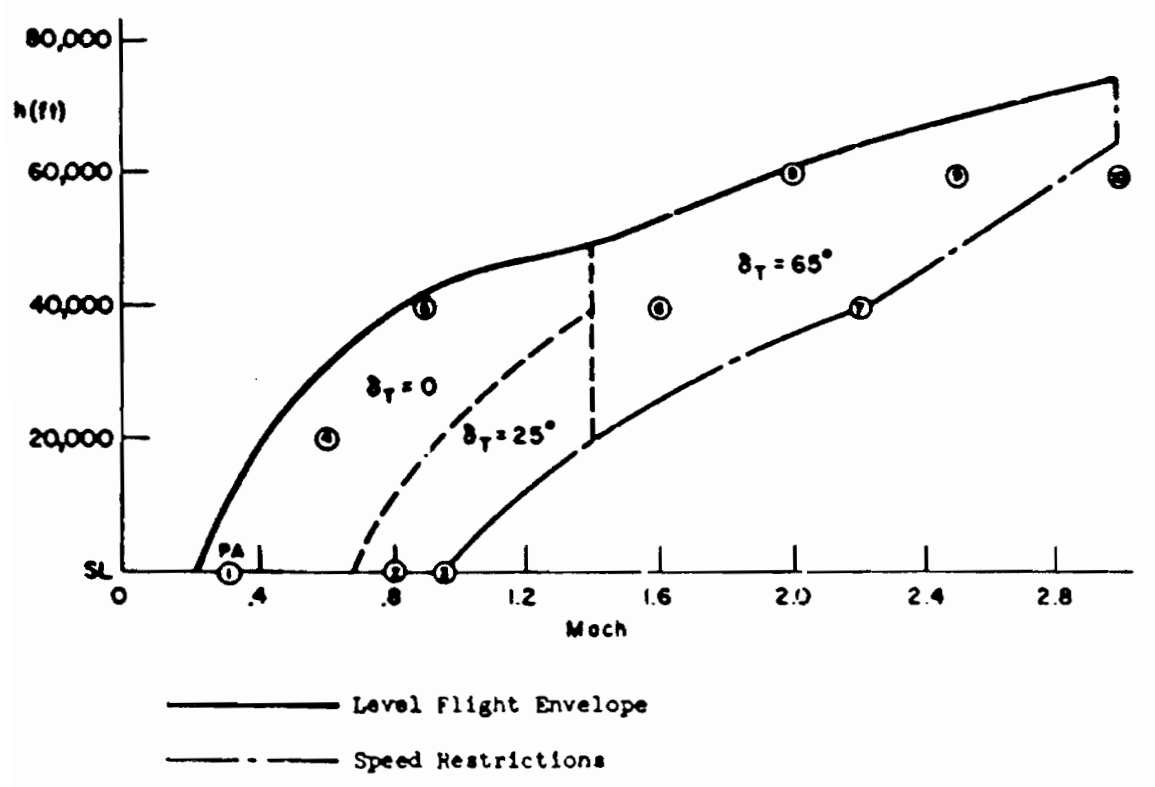

Figure 1-b: $X B-70$ Flight Envelope 
the $X B-70$ is capable of nearly Mach 3 flight at heights up to $75,000 \mathrm{ft}$. In addition, the flight envelope is divided into partitions according to the wing-tip deflection $\left(\delta_{T}\right)$ schedule utilized in the flight test program. Additional details of the $X B-70$ are contained in Appendix 1.

\subsection{Aerodynamic Mode1}

From the references obtained in the literature review, a database was created and implemented into the MATLAB ${ }^{\text {TM }}$ computer-aided engineering software package. With this database, one can input a flight condition and receive the longitudinal and lateral-directional force and moment coefficients. The canard, elevons, and rudders are included in the database. The database incorporates the XB-70's wing tip schedule and covers the Mach number range of 0.8 to 2.5 . Finally, for convenience, all data and utilization programs were implemented in MATLAB ${ }^{\text {TM }}$ using script files.

Linear longitudinal models were constructed from the database. The flight condition of interest is level cruise at Mach 2.5 and $60,000 \mathrm{ft}$. Appendix 1 gives the matrices associated with the linear model of the bare airframe at this cruise flight condition.

\subsection{Pitch Axis Control System}

The XB-70 control system included a Pitch Axis Control System (PACS) which improved the bare airframe characteristics of the $\mathrm{XB}-70$. Among the MATLAB ${ }^{T M}$ 
utilization files is a program that attaches the $\mathrm{XB}-70$ 's PACS to the state space representation of the bare airframe. Appendix 1 also contains the state space matrices associated with the linear model of the bare airframe augmented with the Pitch Axis Control System.

Figure 2 displays the PACS. The inputs are pilot stick force $\left(F_{C C}\right)$, bobweight normal acceleration $\left(a_{z}^{B}\right)$, and the stability Augmentation system's input $\left(\delta_{e_{s A s}}\right.$, described below). The parameters for the $a_{z}^{B}$ segment of the PACS are dependent upon flight condition as shown by the offset chart. The moment arm $l_{B}$ is $-21.69 \mathrm{ft}$ and the gain $B$ is 10 1b./g for the cruise flight condition. The parameter $I_{B}$ is necessary for the computation of $a_{Z}^{B}$. Equation $(2-1)$ gives the expression for the normal acceleration at a distance 1 from the center of gravity in terms of velocity, angle-ofattack and pitch rate.

$$
\mathrm{a}_{\mathrm{z}}=\mathrm{V}(\dot{\alpha}-\mathrm{q})-1 \dot{\mathrm{q}}
$$

Thus, to compute $a_{Z}^{B}$, let $1=l_{B}$. Additionally, the forward path gain $K$ varies with the $x B-70$ flight envelope. The PACS outputs are the commanded deflections for the elevon and canard control surfaces.

Figure 3 presents the stability Augmentation system (SAS) associated with the PACS. The SAS can be turned on or off by the pilot. The inputs to the sAs are $q, a_{z}^{\prime}$, perturbation in Mach number $(\Delta M)$, and $\delta_{C c}$, the internal 

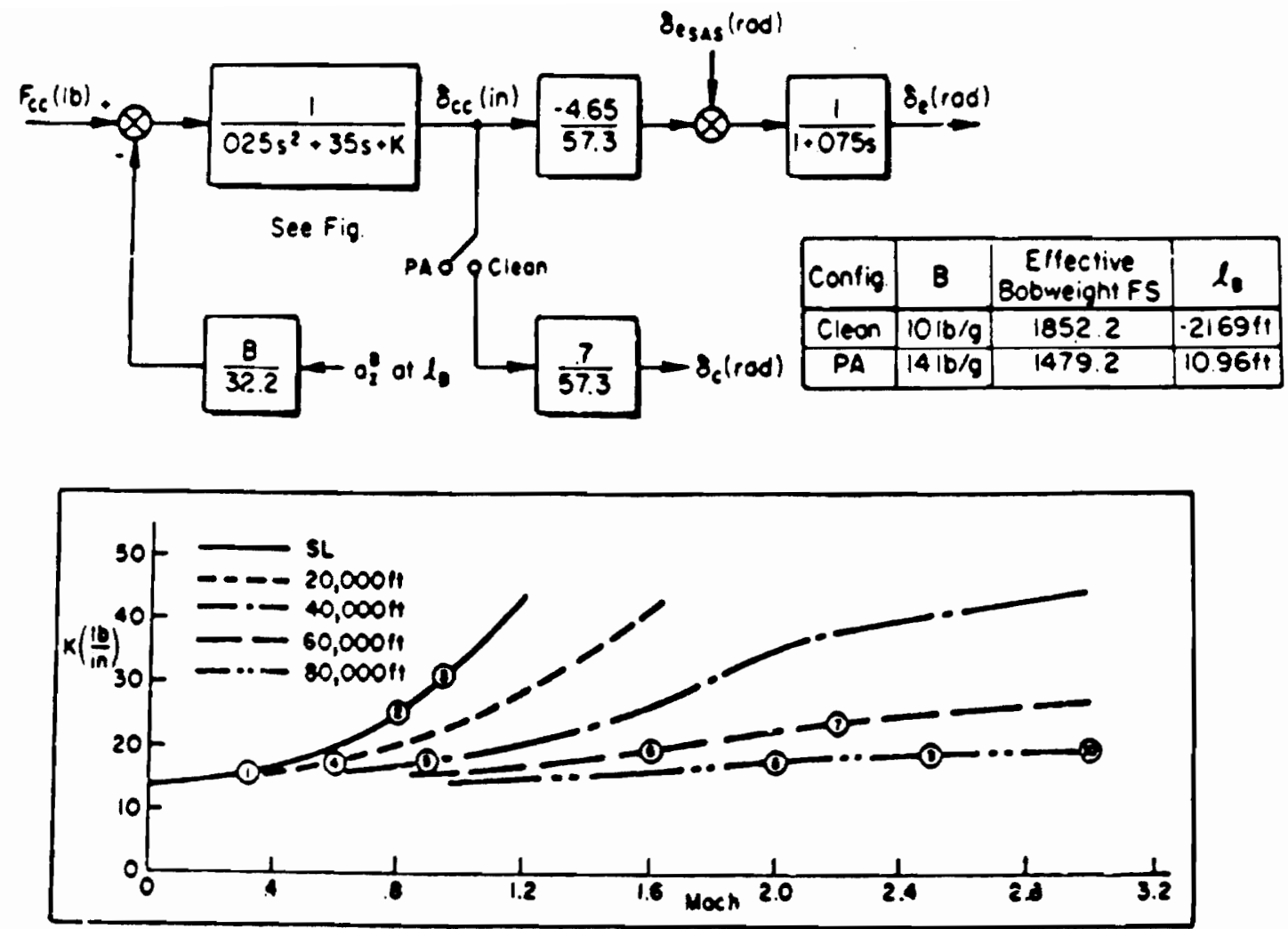

Figure 2: Pitch Axis Control system 


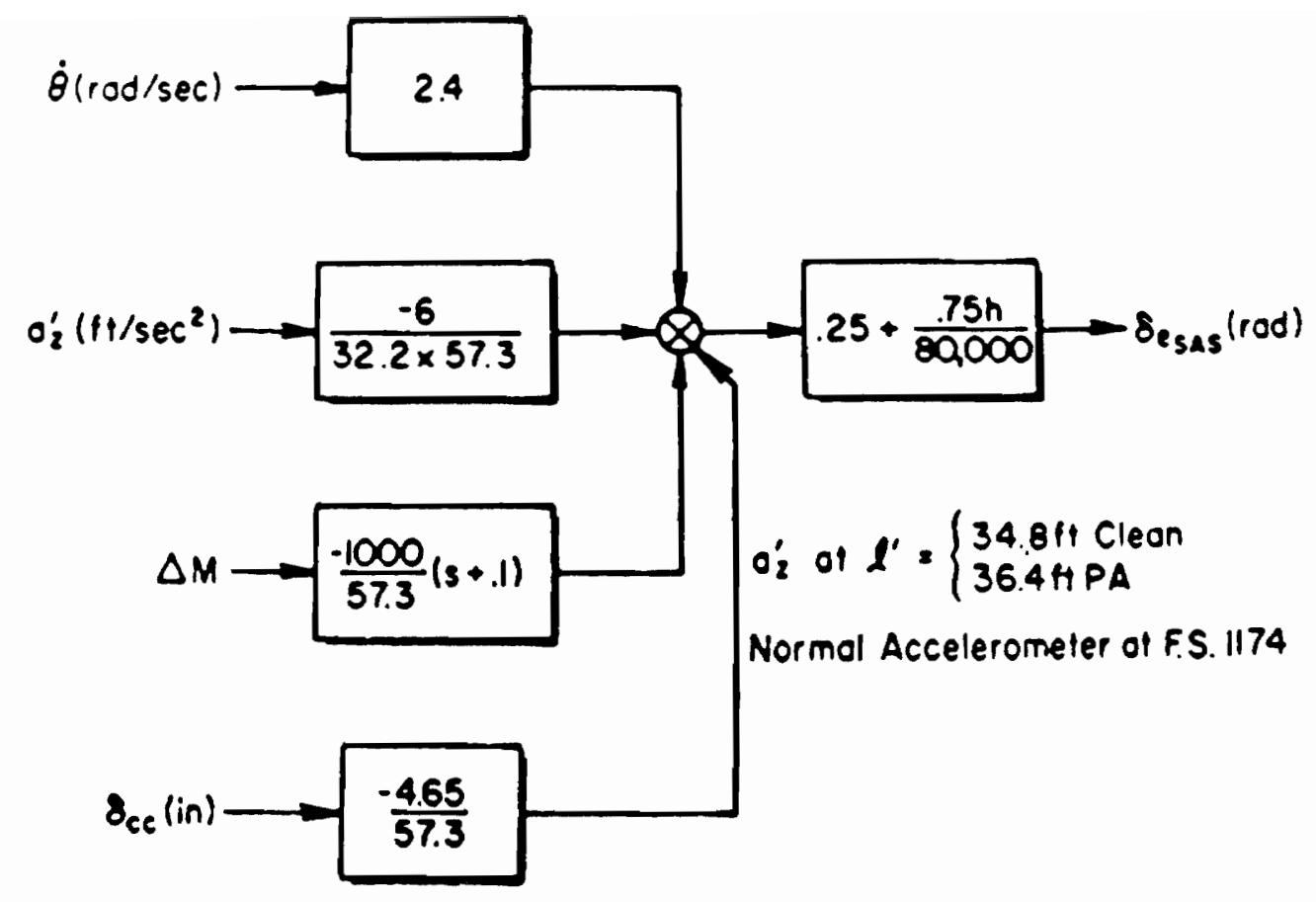

Figure 3: Stability Augmentation System 
PACS variable. These inputs are combined to produce the output $\delta_{e_{\text {sas }}}$ which is then sent to the PACS. The purpose of the SAS is to compensate for the handling qualities deficiencies of the XB-70's bare airframe. The SAS input $a_{Z}^{\prime}$ follows Equation (2-1) where 1 is replaced by $1^{\prime}$. $l^{\prime}$ is 34.8 ft for cruise flight (See Figure 3 ). 


\subsection{Unpiloted Aircraft Dynamic survey}

An investigation of the unpiloted aircraft dynamics will reveal if the poor aircraft handling qualities are the primary source of the altitude excursions. If the aircraft is the source, the aircraft or its control system can be modified to eliminate the problems. If the aircraft is not the source, other suspects, such as pilot-aircraft interactions, need to be analyzed.

To execute the unpiloted aircraft dynamics analysis, the eigenvalues of the phugoid and short period modes are extracted from the linear models of the bare airframe and bare airframe supplemented with the PACS. Next, the damping ratios and natural frequencies are examined. Table 1 shows the longitudinal dynamics at cruise with and without the PACS. As Table 1 portrays, the PACS increases the damping ratios and natural frequencies of the bare airframe modes.

Comparing the dynamics of XB-70 with MIL-STD-1797[19], one can see that the XB-70 meets the necessary conditions for good handling qualities, especially with the PACS on. However, as the XB-70 altitude excursions demonstrate, the MIL-STD-1797 requirements are not sufficient conditions for good handling qualities. To compare with MIL-STD-1797 one usually computes a $4^{\text {th }}$ order equivalent system; however, for the PACS on results shown herein, the necessary parameters are taken directly from the $10^{\text {th }}$ order linear system which 
Table 1: XB-70 Longitudinal Characteristics

$\begin{array}{ccc}\text { Parameter } & \text { PACS off } & \text { PACS On } \\ \zeta_{\mathrm{sp}} & 0.143 & 0.625 \\ { }^{\omega_{\mathrm{sp}}} & 1.722 & 1.965 \\ \zeta_{\mathrm{ph}} & 0.097 & 0.350 \\ \omega_{\mathrm{ph}} & 0.019 & 0.053\end{array}$

Key: $x$ PACS on + PACS off

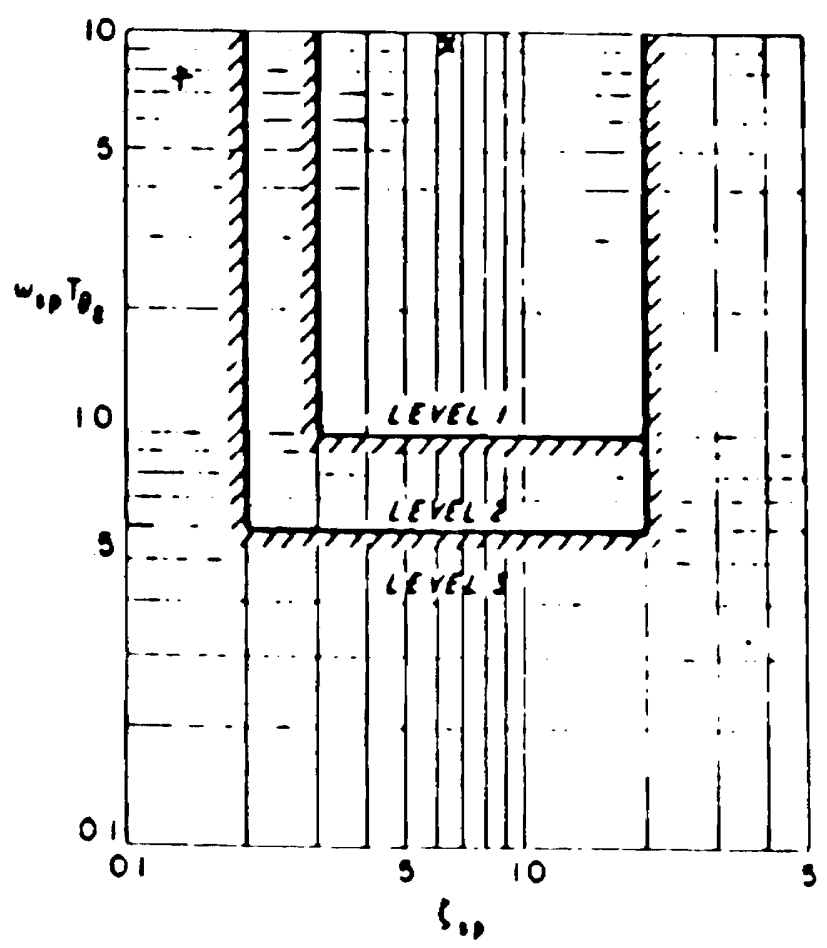

Figure 4: Short-Term Pitch Response to Pitch Controller Requirements 
contains six high frequency poles and four poles that correspond to the effective phugoid and short period modes.

For the PACS-on and -off, Table 1 shows the damping ratios and natural frequencies of the effective short period and phugoid modes. Table 1 shows that the phugoid modes of both cases pass the Category B, Level 1, long-term pitch response requirement which is $\zeta_{\mathrm{ph}}$ must be greater than 0.04 . Figure 4 shows the requirements for short-term pitch response to pitch controller. $\zeta_{s p}$ is on the abscissa and along the ordinate is the product $\omega_{s p} \tau_{\theta_{2}}$. The PACS on case is shown by an $x$ and the PACS off, or bare airframe, case is marked by an + . With the PACS on, the XB-70 lies within the Level 1, Category B region, whereas the bare airframe lies in the Level 3 region.

Figure 5 gives the Category B short-period frequency requirements. The PACS on and off cases are marked as in Figure 4. Along the abscissa is $n / \alpha$ and on the ordinate is $\omega_{\text {sp. As }}$ Figure 5 shows, both cases are in the Level 1 region.

Thus, by examining the XB-70's mode characteristics and comparing them with the necessary requirements of the MILSTD-1797, one would not expect the XB-70 to exhibit unusual behavior. other possible sources of the experienced performance problems must be examined. 


$$
\begin{aligned}
\text { Key: } & \times \text { PACS On } \\
+ & \text { PACS of } f
\end{aligned}
$$

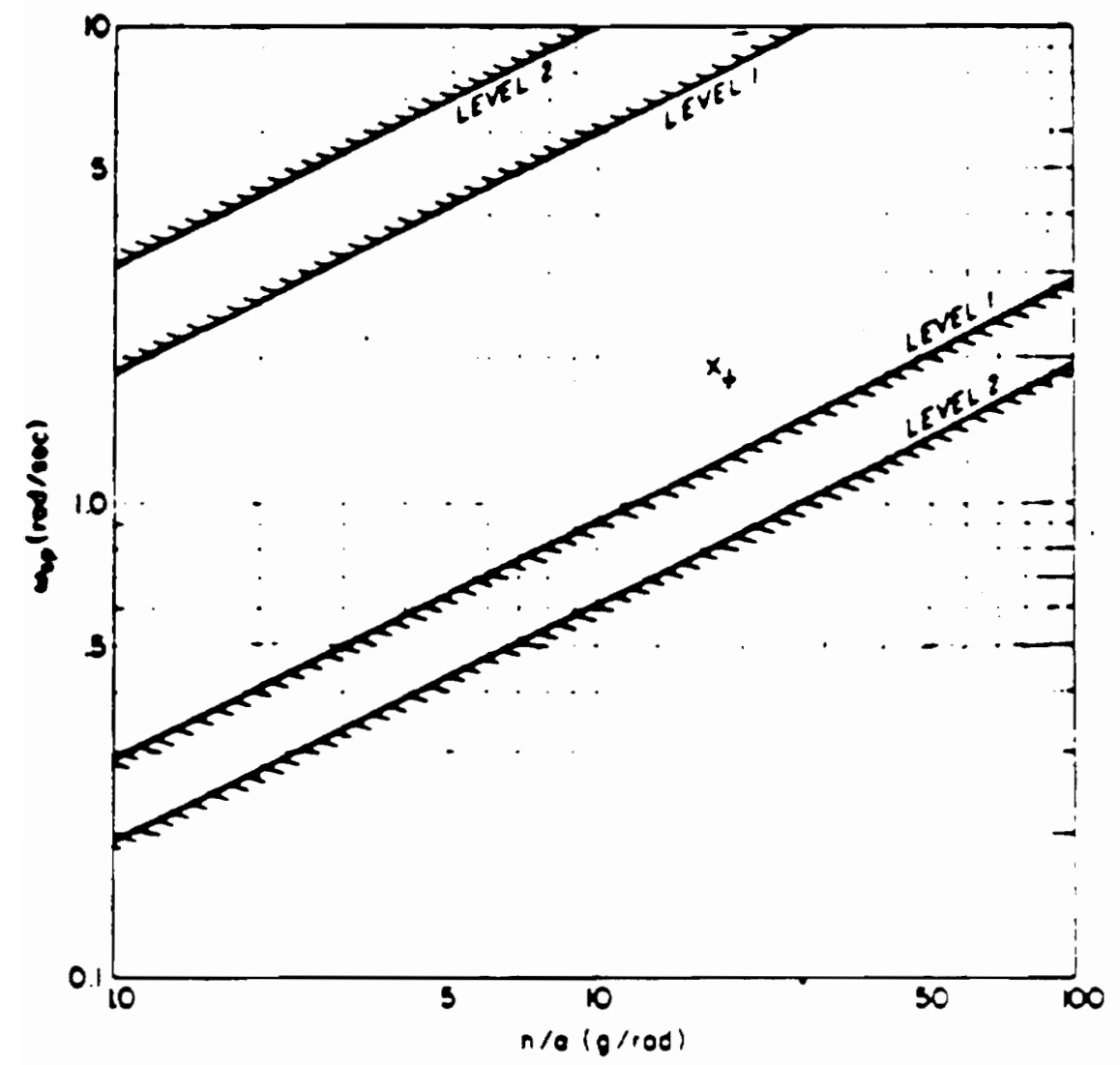

Figure 5: Short-Period Frequency Requirements 


\subsection{Analysis with sequential Pilot Loop closure}

A closer look at the problems encountered in the XB-70 flight test program gives insight to the sources of the altitude excursions. Since $\dot{h}=V s i n(\gamma)$, one degree of flight path angle creates $3000 \mathrm{ft} / \mathrm{min}$ rate of climb at high speed cruise. Thus, precise flight path control is crucial for accurate altitude control at high speed cruise. In analyzing the $\mathrm{XB}-70$ 's handling qualities, Powers reported:

The attitude display system has proved to be inaccurate and inadequate and has not been sufficiently sensitive or responsive for precise instrument flying at high Mach numbers. In addition, there has been an apparent lag in the altitude information. To achieve acceptable altitude control, increased attention had to be devoted to the longitudinal control task, which was reflected in the "acceptable but unsatisfactory" ratings for this flight regime[1]. Moreover, Wolowicz and Yancey reported that:

More pilot concentration was necessary at the higher supersonic Mach numbers to avoid undesirable changes in altitude. Inadequacies in the pilot's display contributed to this need for greater pilot concentration. One deficiency was 
the low resolution of the attitude display. which

the pilots used for altitude control[2].

The inadequate displays and sensors resulted in pilot induced altitude excursions ranging in magnitude from $300 \mathrm{ft}$ to $800 \mathrm{ft.[2]}$. To alleviate these problems, engineers doubled the attitude display's resolution and improved the attitude sensor. The XB-70 experience apparently demonstrates that severe control problems can afflict high speed aircraft even if the bare airframe seemingly has good handling characteristics.

This chapter examines the problem from a classical control theory viewpoint to determine the root loci trends for various feedback structures. From the root loci trends, an appropriate pilot control strategy is developed.

Much research on manual control has been conducted involving general and specific pilot loop closure structures, from which acceptable control techniques have been predicted[20]. Figure 6 presents Craig and Heffley's work with control technique formulation and loop closure structure for conventional and unconventional landing control schemes. For example, Figure 6-a shows the pilot loop closure block diagram associated with the unconventional 'powered lift' short field landing technique. In this technique, the pilot controls $\dot{h}$ with thrust and $u$ with the inner attitude loop. Figure 6-b presents the loop 


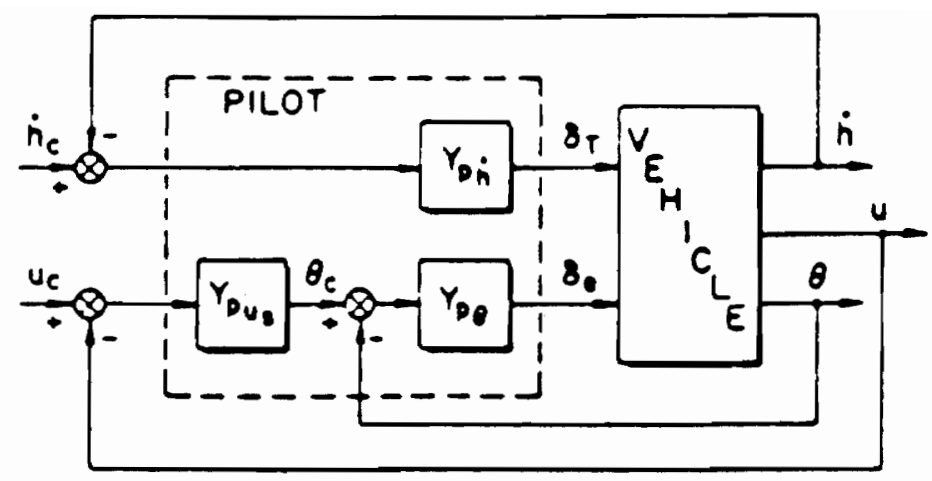

Figure 6-a: Short Field Landing Technique

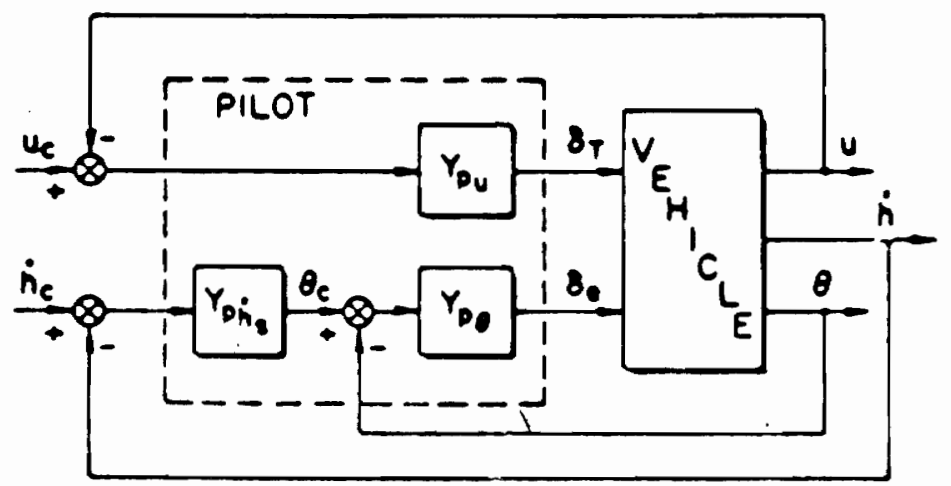

Figure 6-b: Conventional Control Technique 
closure block diagram associated with the conventional control technique. This technique requires the pilot to control $u$ with thrust and $\dot{h}$ with the inner attitude loop. For both approach and landing techniques, the inner attitude loop is essential. However, the requirements on the inner loop qualities, such as bandwidth, depend upon the outer loop structure[20].

The conventional control technique is appropriate for the cruise flight condition investigated in this research. The pilot loop closure assumes that $\theta$ and $\dot{h}$ are available to control the aircraft. As noted above, $\dot{h}=V \sin (\gamma)$; thus for constant $\mathrm{V}$ and $\operatorname{small} \gamma, \dot{\mathrm{h}}$ control may be executed using precise control of $\gamma$. The analysis utilizes the transfer functions $\gamma /$ FCC and $\theta / F C C$ for the cruise flight condition with the SAS on. The transfer functions are created using the linear system and the MATLAB ${ }^{T M}$ function ss2tf which performs state space to transfer function conversion. The pilot is approximated as proportional gains $\left(\mathrm{K}_{\theta}, \mathrm{K}_{\gamma}\right)$. These gains are varied to explore the root loci trends. The trends reveal how the short period and phugoid modes are affected by each feedback loop. First, the trends associated with closing the attitude loop without the flight path loop are examined. With $k_{y}$ equal to zero, this is like taking flight path information away from the pilot. Then the trends resulting from closing the flight path loop 
without the attitude loop are studied. With $\mathrm{K}_{\theta}$ equal to zero, this is equivalent to taking away attitude information from the pilot. The trends for flight path only feedback are analogous to the $\mathrm{XB}-70$ experience because the inadequate displays prevented the pilot from properly closing the inner attitude loop. Then, sequential loop closure is executed with flight path loop closure after pitch attitude closure. Lastly, the trends for sequential loop closure and flight path only feedback are compared. The comparison shows the necessity of the inner attitude loop to provide phugoid stability.

\subsection{Pitch Angle Loop Closure}

The closed loop poles of the pitch attitude loop are the roots of the characteristic equation:

$$
1 \pm \mathrm{K}_{\theta}(\theta / \mathrm{FCC})=0
$$

The + signifies negative feedback and the - signifies positive feedback. $\quad K_{\theta}$ is defined as greater than or equal to zero. A root locus plot gives the pole locations associated with Equation (4-1) over a wide range of $\mathrm{K}_{\theta}$ values. In a root locus plot, the poles' imaginary component is on the ordinate and the poles' real component is along the abscissa. Positive $\mathrm{K}_{\theta}$ variation pole migration is indicated by an arrow. The poles associated with zero gain are the open loop poles and are marked with an $x$. 


\subsubsection{Negative Pitch Angle Feedback}

Figures 7 and 8 show the root loci for negative pitch angle feedback. Although the aircraft and PACS result in a $10^{\text {th }}$ order system, only the roots associated with the effective phugoid and short period modes are isolated and shown in Figures 7 and 8 . Figure 7 shows the phugoid mode results and Figure 8 gives the short period mode results.

For the phugoid mode, negative feedback increases the damping ratio. The complex conjugate phugoid poles split as $\mathrm{K}_{\theta}$ increases; however, due to $\mathrm{a}$ zero at -0.035 , both branches remain stable, i.e. the real component of the poles are always less than zero. Unlike the phugoid mode, the short period mode becomes unstable once $\mathrm{K}_{\theta}$ becomes sufficiently large.

In summary, the negative feedback root loci plots reveal that as $k_{\theta}$ increases, $\zeta_{\text {ph }}$ increases while $\zeta_{\text {sp }}$ decreases. Thus, negative pitch angle feedback has a destabilizing effect on the short period mode and a stabilizing effect on the phugoid mode.

\subsubsection{Positive Pitch Angle Feedback}

The root loci for positive pitch angle feedback are given by Figures 9 and 10. Figure 9 shows the results for the phugoid mode while Figure 10 displays the results for the short period mode. 


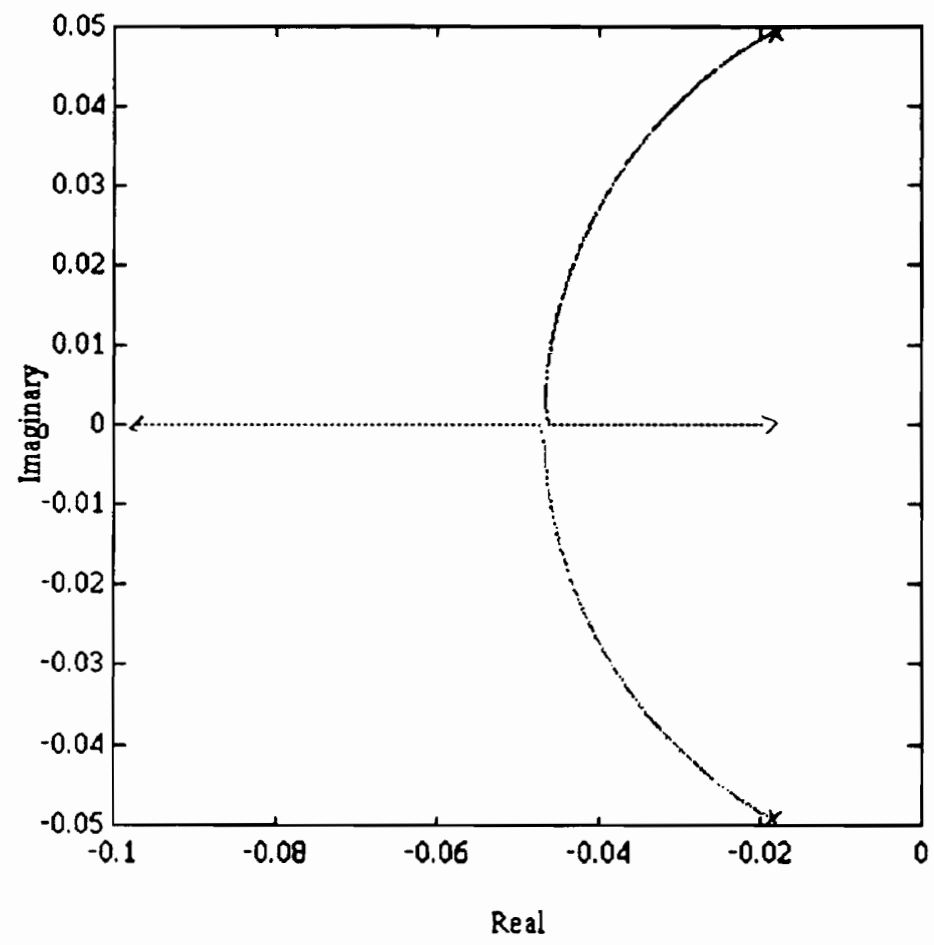

Figure 7: Attitude Closure: Negative Feedback, Phugoid Mode Root Locus 


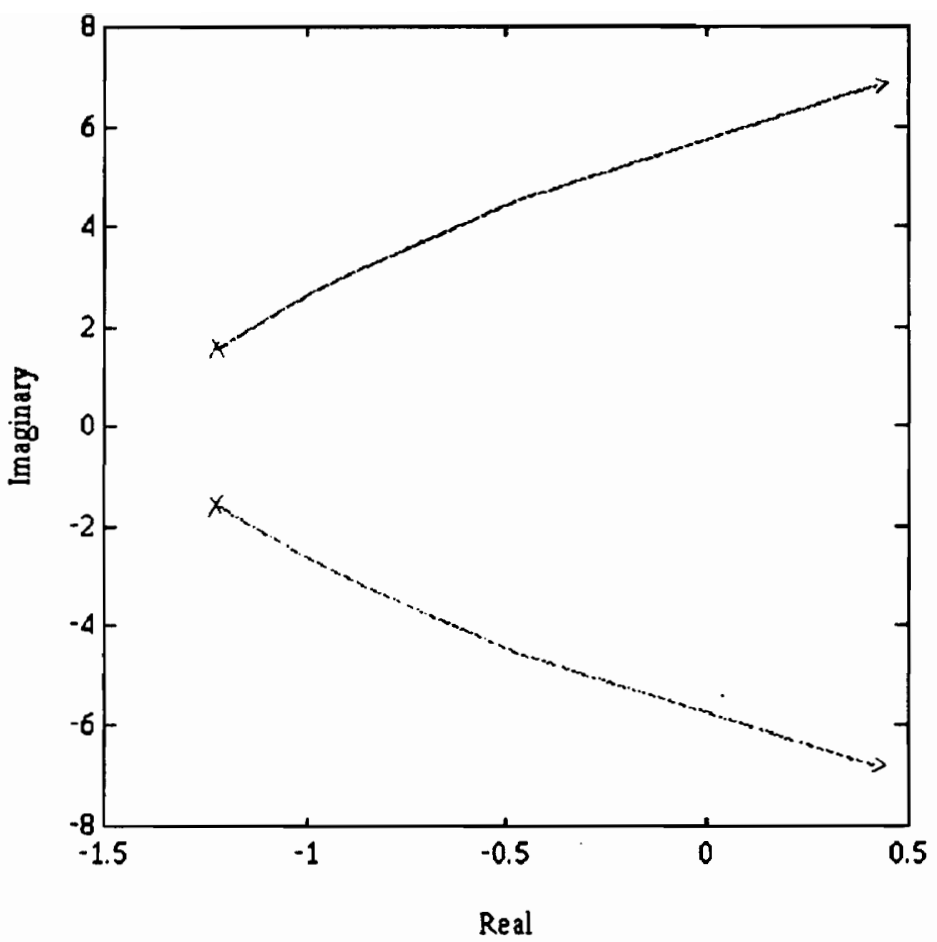

Figure 8: Attitude Closure: Negative Feedback, Short Period Mode Root Locus 


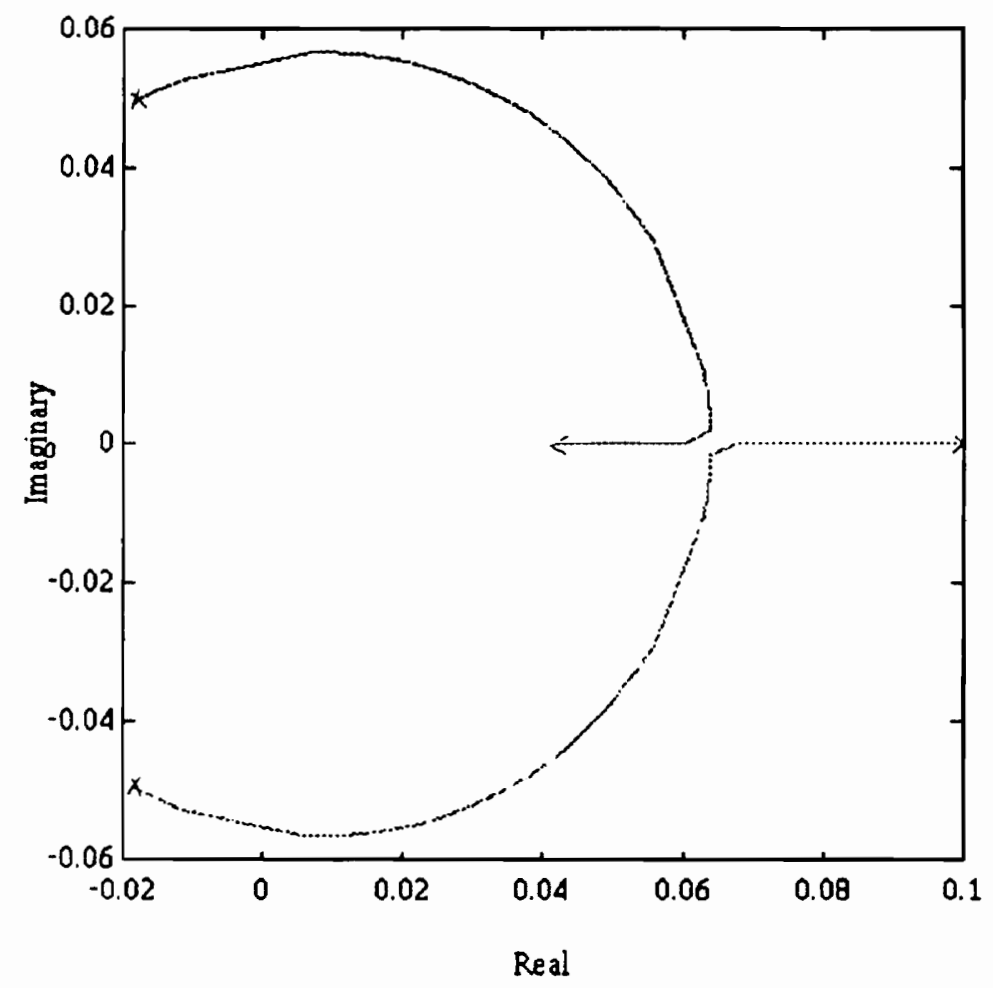

Figure 9: Attitude Closure: Positive Feedback, Phugoid Mode Root Locus 


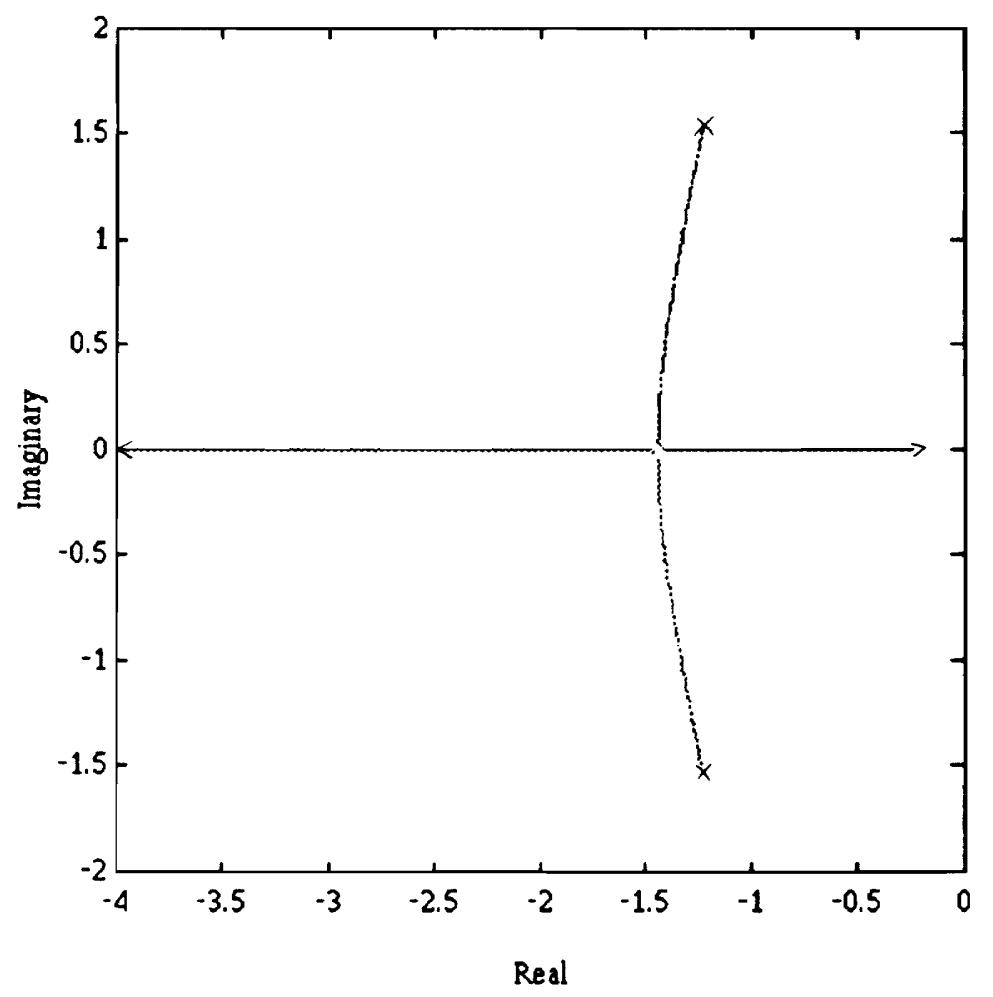

Figure 10: Attitude Closure: Positive Feedback: Short Period Mode Root Locus 
From these figures, one can see that positive feedback affects the modes in the opposite manner by which negative feedback does. Thus, as $\mathrm{k}_{\theta}$ increases for positive feedback, $\zeta_{\mathrm{ph}}$ decreases and $\zeta_{\mathrm{sp}}$ increases. Therefore, positive pitch angle feedback has a destabilizing effect on the phugoid mode but has a stabilizing effect on the short period mode for moderate gain values.

4.2 Flight Path Angle Loop Closure

Next, the flight path angle loop is examined. The closed loop poles are the roots of the following characteristic equation.

$$
1 \pm \mathrm{K}_{\gamma}(\gamma / \mathrm{FCC})=0
$$

Again, the gain is greater than or equal to zero and \pm represents negative and positive feedback, respectively. 4.2.1 Negative Flight Path Feedback

The root loci plots for negative flight path feedback are given in Figures 11 and 12. Figure 11 gives the phugoid mode results and Figure 12 presents the short period mode results. From Figure 11, one can see that the phugoid mode damping ratio increases and the mode splits into two real poles. From Figure 12, the short period mode damping ratio decreases and the mode becomes unstable. Summarizing, the figures show that as $\mathrm{k}_{\mathrm{y}}$ increases, $\zeta_{\mathrm{sp}}$ decreases and $\zeta_{\mathrm{ph}}$ increases. Thus, similar to negative attitude feedback, negative flight path feedback has a stabilizing effect on 


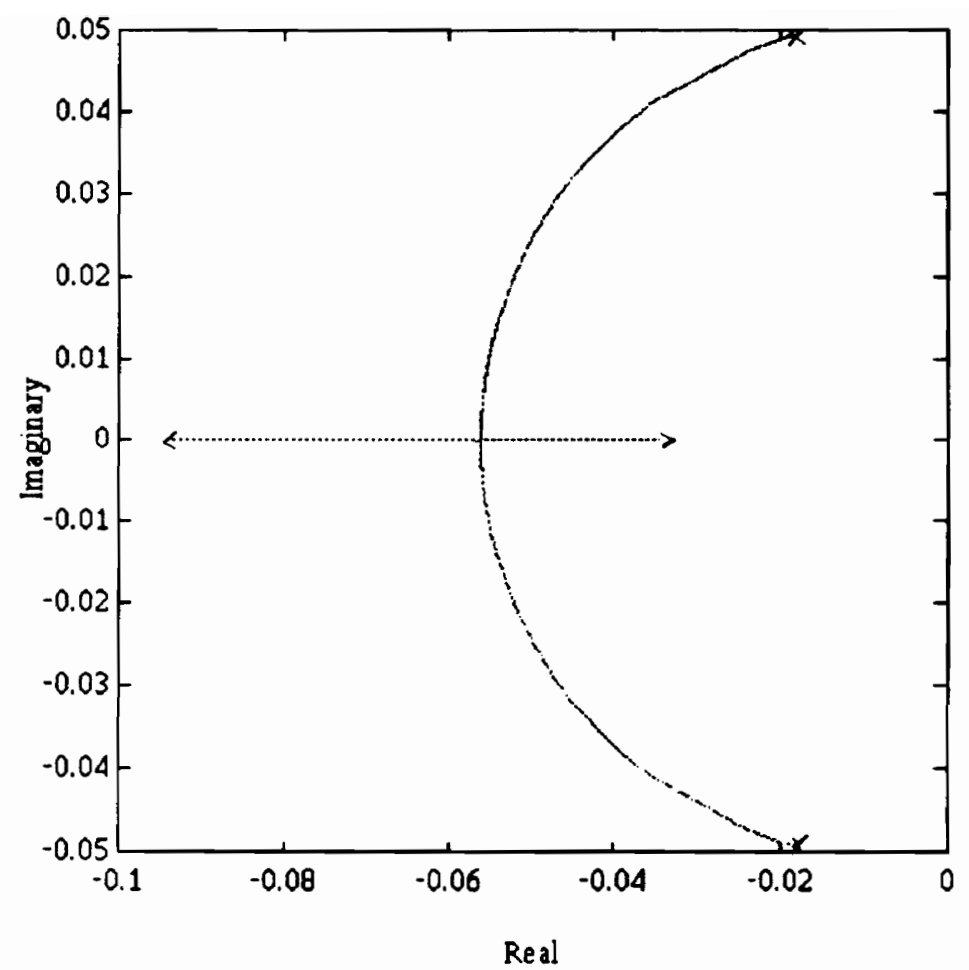

Figure 11: Flight Path Closure: Negative Feedback, Phugoid Mode Root Locus 


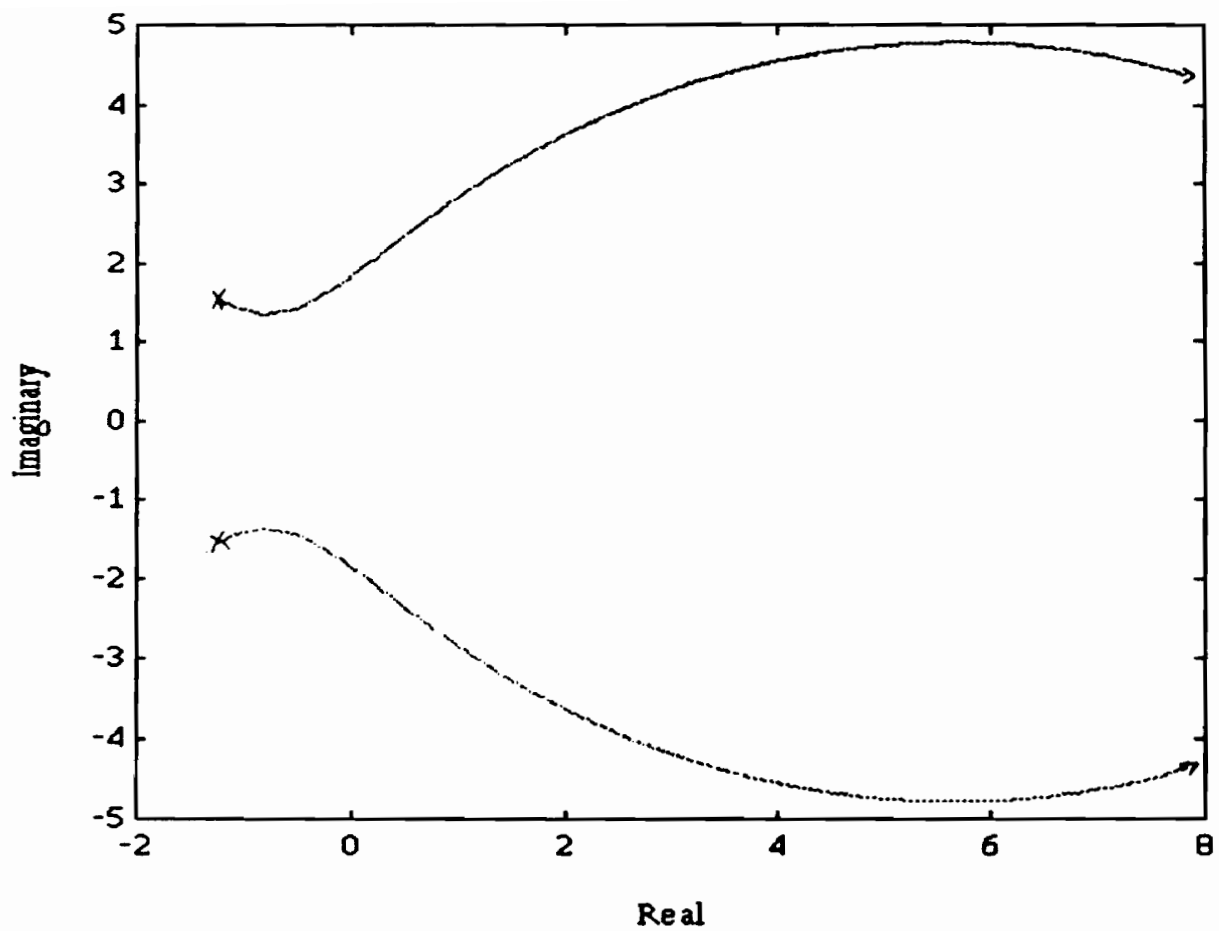

Figure 12: Flight Path Closure: Negative Feedback, Short Period Mode Root Locus 
the phugoid and a destabilizing effect on the short period mode.

\subsubsection{Positive Flight Path Feedback}

The phugoid and short period modes' root loci for positive flight path feedback are given in Figures 13 and 14, respectively. From Figure 13, one can see that the phugoid mode destabilizes, then splits, and finally, one branch moves toward the left-half-plane (LHP) while the other moves farther into the right-half-plane (RHP). In Figure 14, the short period mode damping ratio increases but later decreases and finally the mode becomes unstable with large $k_{\gamma}$. Summarizing, the figures show that for moderate values of $\mathrm{k}_{\mathrm{y}}, \zeta_{\mathrm{sp}}$ increases while $\zeta_{\mathrm{ph}}$ decreases.

\subsection{Sequential Loop Closure}

Finally, the aforementioned loops are combined in order to study the trends of sequential loop closure implied by the pilot structure presented in Figure 6-b. First, the pitch loop is closed and then the flight path loop is closed. From the results of sections 4.1 and 4.2 , it would appear that two feasible closure options exist. These options are:

1. To employ outer negative flight path angle feedback with inner positive pitch angle feedback.

2. To employ inner negative pitch angle feedback and outer positive flight path angle feedback. 


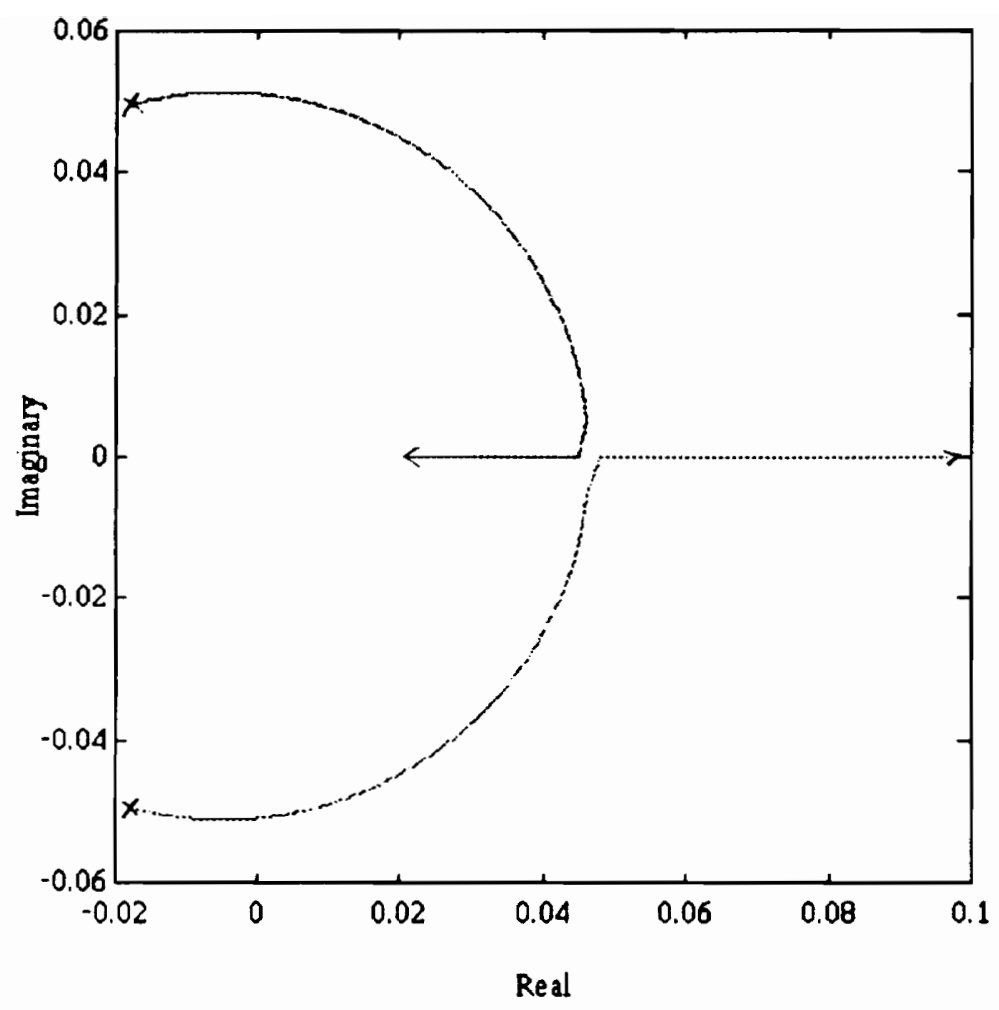

Figure 13: Flight Path Closure: Positive Feedback, Phugoid Mode Root Locus 


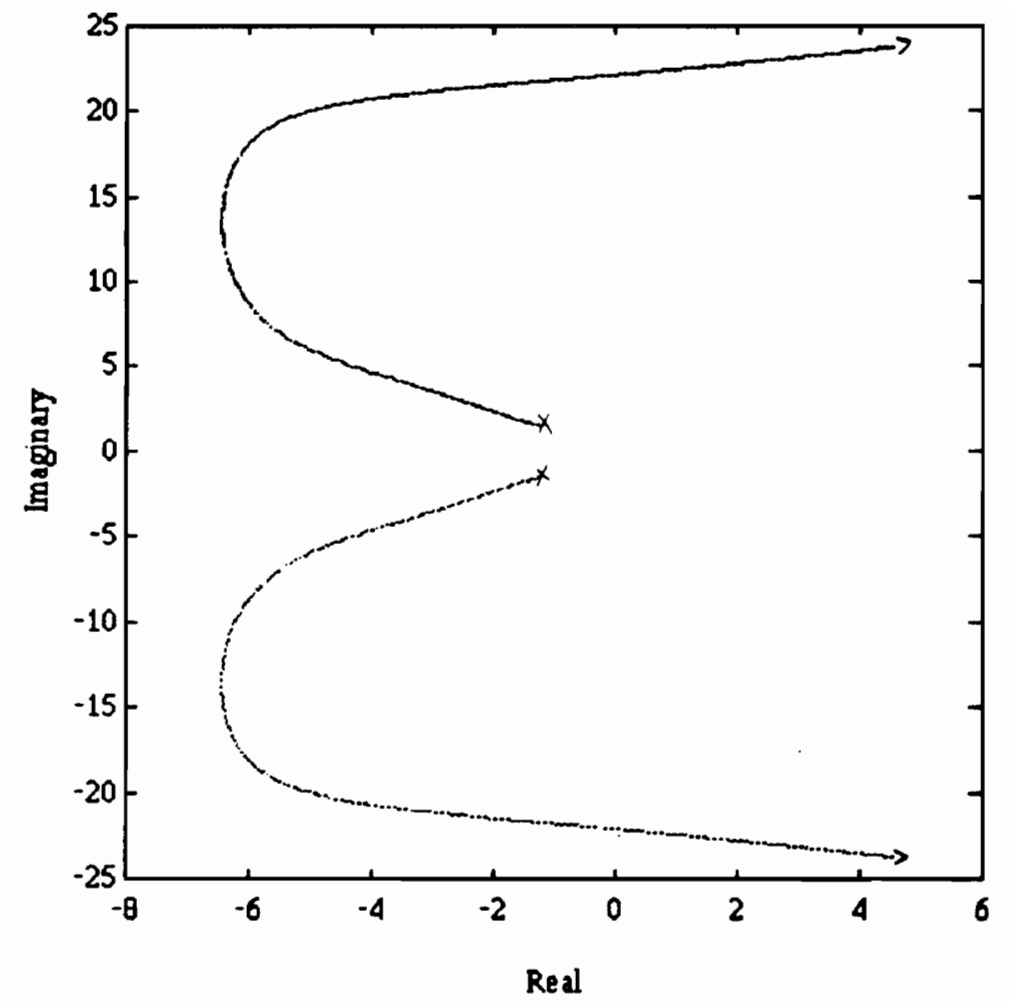

Figure 14: Flight Path Closure: Positive Feedback, Short Period Mode Root Locus 
These are the feasible options because, in each case, one of the loops improves phugoid damping ratio (while decreasing short period damping ratio); the loop that performs this is listed first in each option. The other loop improves short period damping ratio (while decreasing phugoid damping ratio); this loop is listed second. Thus the tradeoffs must be analyzed to determine the best option. The 'natural' control scheme would be to control the short period mode with the attitude loop and the phugoid mode with the outer flight path loop. However, an examination of Figures 7 through 14 reveals that option 2 is, in fact, a better choice because it provides higher damping ratios and natural frequencies. A higher closedloop effective $\omega_{\text {sp }}$ allows the pilot to more effectively control flight path angle and $\dot{h}$. Figure 14 shows that positive flight path feedback increases the $\zeta_{s p}$ and $\omega_{s p}$, whereas positive pitch feedback (Figure 10) increases $\zeta_{s p}$ but decreases $\omega_{\text {sp }}$. A similar discussion applies to the phugoid mode. Overall, option 2 will have better phugoid and short period performance than option 1; therefore, option 2 is examined for sequential loop closure.

with Option 2, closing the pitch angle loop with negative feedback improves phugoid stability. Since the positive flight path feedback can destabilize the phugoid mode, the inner loop closure should allow a greater range of 
$\mathrm{K}_{\gamma}$ possibilities. To test this hypothesis, a value of $6 \mathrm{~dB}$ was chosen for $K_{\theta}$ and the inner loop was closed with negative feedback.

The poles of the sequential loop closure for option 2 are computed from the characteristic equation:

$$
1-\mathrm{K}_{\gamma}(\gamma / \text { FCC })+\mathrm{K}_{\theta}(\theta / \text { FCC })=0 \quad(4-3),
$$

where $k_{\theta}$ is $6 d B$ and $k_{y}$ is greater than or equal to zero. Once the closed loop poles are computed, the damping ratio and natural frequency are calculated.

\subsubsection{Short Period Mode Results}

Table 2 presents the results for sequential loop closure $(\gamma+\theta)$ and flight path only closure $(\gamma)$ in terms of the parameters $\zeta_{s p}$ and $\omega_{s p}$. Table 2's comparison between $\zeta_{\gamma}$ and $\zeta_{\gamma+\theta}$ shows that the $(\gamma)$ case has better damping; however, as $k_{\gamma}$ increases the two short period modes have practically the same characteristics. On the other hand, the values of $\omega_{\gamma+\theta}$ are consistently greater than $\omega_{\gamma}$ i this is attributable to the closed inner attitude loop. Thus, for a particular $k_{\gamma}$, the $(\gamma+\theta)$ case yields better flight path performance than the $(\gamma)$ case. In addition, for a given lower bound on $\omega_{\mathrm{sp}}$, the $(\gamma+\theta)$ case requires less $\mathrm{K}_{\gamma}$ which leads to lower control power requirements or pilot workload. 4.3.2 Phugoid Mode Results

Table 3 compares the results of flight path closure $(\gamma)$ and sequential loop closure $(\gamma+\theta)$ by examining the 
Table 2: Short Period Mode Variation with Outer Loop Gain

$\begin{array}{ccccc}\mathrm{K}_{\gamma}(\mathrm{dB}) & \omega_{\gamma} & \omega_{\gamma+\theta} & \zeta_{\gamma} & \zeta_{\gamma+\theta} \\ \text {-Open Loop- } & 1.9649 & 2.1478 & 0.6246 & 0.5467 \\ -16.0270 & 1.9674 & 2.1497 & 0.6248 & 0.5471 \\ -6.3613 & 1.9725 & 2.1535 & 0.6253 & 0.5477 \\ 3.2572 & 1.9878 & 2.1650 & 0.6266 & 0.5497 \\ 7.7304 & 2.0028 & 2.1765 & 0.6278 & 0.5515 \\ 10.6685 & 2.0177 & 2.1879 & 0.6289 & 0.5533 \\ 14.8806 & 2.0490 & 2.2124 & 0.6311 & 0.5569 \\ 24.0899 & 2.1889 & 2.3260 & 0.6379 & 0.5706 \\ 48.5842 & 3.7888 & 3.8299 & 0.6442 & 0.6183 \\ 57.8949 & 5.5871 & 5.5962 & 0.6517 & 0.6361 \\ 63.7145 & 7.9338 & 7.8984 & 0.6390 & 0.6295 \\ 66.8926 & 9.8439 & 9.8014 & 0.5903 & 0.5850 \\ 69.3208 & 11.4986 & 11.4643 & 0.5368 & 0.5333 \\ 70.6898 & 12.5280 & 12.4987 & 0.5035 & 0.5006\end{array}$

Table 3: Phugoid Mode Variation with outer Loop Gain

\begin{tabular}{ccccc}
$\mathrm{K}_{\gamma}(\mathrm{dB})$ & $\omega_{\gamma}$ & $\omega_{\gamma+\theta}$ & $\zeta_{\gamma}$ & \multicolumn{1}{c}{$\zeta_{\gamma+\theta}$} \\
-Open LoOp- & 0.0527 & 0.0495 & 0.3495 & 0.6997 \\
-21.0100 & 0.0526 & 0.0494 & 0.3305 & 0.6831 \\
-10.5524 & 0.0524 & 0.0492 & 0.2861 & 0.6442 \\
-0.1822 & 0.0517 & 0.0487 & 0.1396 & 0.5159 \\
1.0432 & 0.0515 & 0.0485 & 0.1076 & 0.4879 \\
3.2515 & 0.0512 & 0.0483 & 0.0371 & 0.4260 \\
3.4270 & 0.0512 & 0.0482 & 0.0307 & 0.4204 \\
3.9331 & 0.0511 & 0.0482 & 0.0114 & 0.4034 \\
4.2548 & 0.0510 & 0.0481 & -0.0014 & 0.3922 \\
11.3858 & 0.0489 & 0.0464 & -0.4569 & -0.0089
\end{tabular}

Key:

$\left(\omega_{\gamma}, \zeta_{\gamma}\right):$ Flight Path closure Results (Section 4.2) $\left(\omega_{\gamma+\theta}, \zeta_{\gamma+\theta}\right)$ : Sequential Loop closure Results (Section 4.3) 
parameters $\zeta_{\mathrm{ph}}$ and $\omega_{\mathrm{ph}}$. Table $3^{\prime}$ 's results show that $\omega_{\gamma+\theta}$ is slightly lower than $\omega_{\gamma} ;$ however, the damping is significantly higher for the $(\gamma+\theta)$ case. Thus, for a given lower bound on $\zeta_{\mathrm{ph}}$, a higher value of $k_{\gamma}$ is permitted for the $(\gamma+\theta)$ case compared to the $(\gamma)$ case. This translates into higher possible $\omega_{\text {sp }}$ and thus better tracking performance for the $(\gamma+\theta)$ case.

\subsubsection{Sequential Loop Closure Discussion}

The feedback trends presented in Tables 2 and 3 prove the hypothesis that the inner attitude loop closure allows a greater range of useful $k_{\gamma}$. The value of $k_{\gamma}$ for which the phugoid destabilizes more than doubles (almost triples in dB) with the closure of the inner attitude loop. The short period mode for the $(\gamma+\theta)$ case begins with lower damping than the $(\gamma)$ case, but Figure 4 shows the difference is not significant with respect to the MIL-STD-1797 handling qualities requirements. In addition, $\omega_{s p}$ is increased by a significant amount.

The phugoid mode stability is greatly improved under sequential loop closure. The inner attitude loop closure is responsible for increasing the open loop damping ratio over 100 percent compared to the $(\gamma)$ case. This is very important because, as Table 3 shows, the $\omega^{\omega} \mathrm{ph}$ and $\zeta_{\mathrm{ph}}$ decrease at about the same rate for both cases. This fact places added importance to the location of open loop poles. 
Furthermore, a typical $\omega_{\text {sp }}$ range for tracking flight path is 2 to $4 \mathrm{rad} / \mathrm{sec}$. Comparing Tables 2 and 3 , one can see that as $K_{\gamma}$ increases, the $\omega_{\text {sp }}$ increases. To maximize short period performance, the 'proportional pilot' would prefer to increase $K_{\gamma}$ to near $55 \mathrm{~dB}$, but it cannot due to the phugoid instability constraint. For the $(\gamma)$ case, $\omega_{\text {sp }}$ reaches $2 \mathrm{rad} / \mathrm{sec}$ as $\zeta_{\mathrm{ph}}$ goes to zero. On the other hand, with the $(\gamma+\theta)$ case, $\omega_{s p}$ is in the range at $k_{\gamma}$ equal zero and continues to climb to $2.19 \mathrm{rad} / \mathrm{sec}$ before the phugoid destabilizes.

Therefore, a comparison of the sequential loop closure and flight path only feedback trends reveal that the inner attitude loop is crucial for phugoid mode stability. The enhanced stability allows greater short period natural frequency which improves flight path tracking. Thus, without the inner loop, the pilot's ability to control flight path, and thus rate of climb, is degraded.

The proportional gain analysis reveals that an appropriate pilot control strategy for the XB-70 would be to use an inner loop with negative attitude feedback to control phugoid dynamics and an outer loop with positive flight path feedback to improve short period mode dynamics. As noted in Chapter 1, the XB-70 test pilots experienced difficulty in achieving acceptable performance. This behavior was not due to the airframe dynamics, but rather attributable to 
inadequate sensors and displays, in particular those associated with pitch information. The inadequate equipment prevented the pilots from properly closing the inner attitude loop. As the root loci trends have shown, the inner loop is necessary for phugoid damping. Thus, with the XB-70, altitude excursions occurred due to the low damped phugoid mode characteristics that result when only the flight path loop was closed by the pilot using the rate-ofclimb display. 


\subsection{Analysis with Modern Control Methods}

This chapter discusses the application of modern control theory to investigate the piloting problems associated with high speed cruise.

\subsection{Pilot Modeling}

Once the aerodynamic modeling and the sequential loop closure analysis had been completed, it was of interest to see if a modern control theory analysis would predict the same pilot/vehicle interaction. In 1970, Kleinman, Baron, and Levison developed the Optimal Control Model (OCM) of the human operator, which is based on a time domain optimal control methodology. The ocM assumes a well motivated, well-trained pilot will behave optimally in some fashion, compensating for task and vehicle. The validity of the ocM has been proven under many applications[21].

Figure 15 displays the OCM block diagram. The OCM receives a vector of pilot observations ( $y$ ). The observations are time delayed and noise is added. A Kalman filter is used to compute estimates of the delayed states. The estimates are passed through a least-mean-squares predictor to provide lead equalization. The model employs a linear quadratic solution for pilot gains. A first order lag is used to represent neuromotor dynamics.

In addition, the OCM accounts for attention allocation and perception threshold effects. Equation (5-1) gives the 


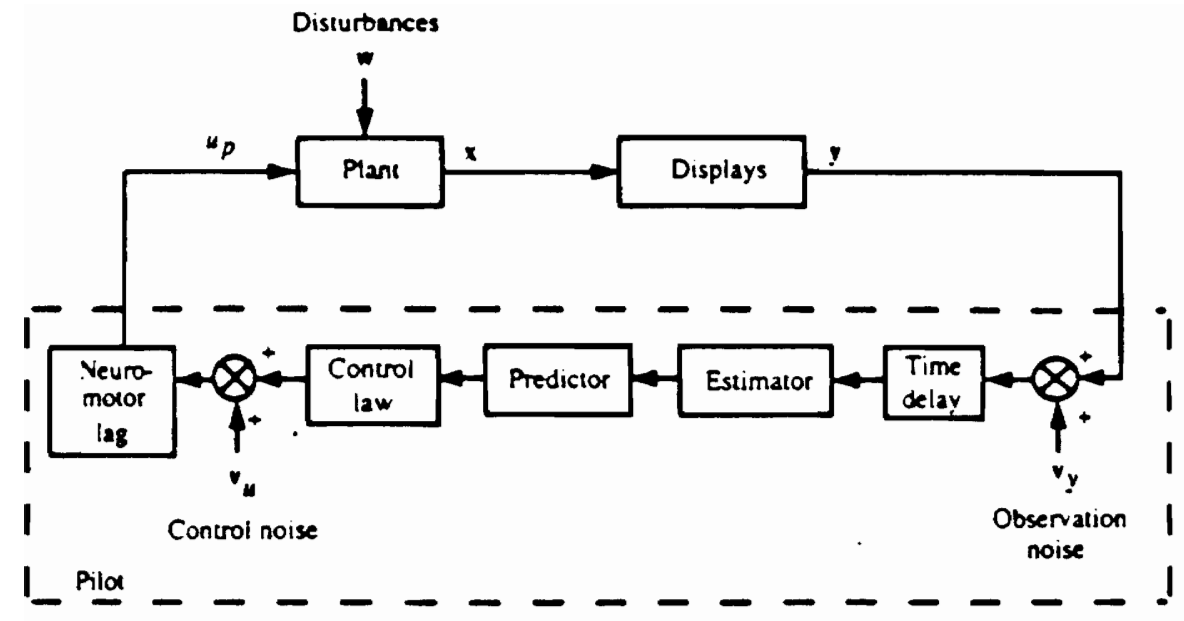

Figure 15: Block Diagram of OCM

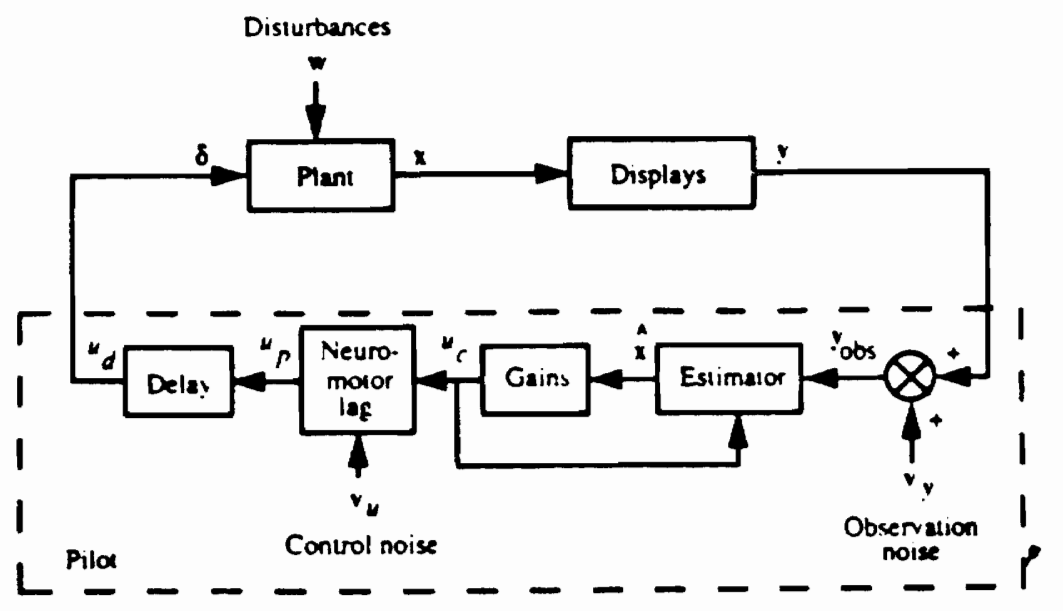

Figure 16: Block Diagram of MOCM 
relationship between pilot observation noise and pilot observation thresholds[22].

$$
\mathbf{v}_{\mathbf{y}_{i}}=\frac{\pi \rho_{\mathbf{y}_{i}}}{\mathbf{f}_{\mathbf{y}_{i}}} \frac{\sigma_{\mathbf{y}_{i}}^{2}}{\left[1-\operatorname{erf}\left\{\mathbf{a}_{\mathrm{TH}_{i}} /\left(\sqrt{2} \sigma_{y_{i}}\right)\right\}\right]^{2}}
$$

Equation (5-1) shows that the noise contaminating the $i^{\text {th }}$ observation is dependent upon the $i^{\text {th }}$ observation's signalto-noise ratio $\left(\rho_{Y_{i}}\right)$, observation variance $\left(\sigma_{Y_{i}}\right)$, attention allocation $\left(f_{Y_{i}}\right)$, and threshold $\left(a_{\mathrm{TH}}\right)$. The denominator of Equation (5-1) models the influence of attention allocation and thresholds. Lower attention allocation and higher thresholds increase the observation noise. The erf() is the error function defined by Equation (5-2)[23].

$$
\operatorname{erf}(x)=\frac{2}{\sqrt{\pi}} \int_{0}^{x} e^{-\xi^{2}} d \xi
$$

The pilot's control objective is modeled by a linear quadratic solution that minimizes the cost function given by Equation $(5-3)$ :

$$
J_{p}=E_{\infty}\left\{\mathbf{y}^{\mathrm{T}} \mathbf{Q}_{\mathbf{y}} \mathbf{y}+\mathrm{u}_{\mathrm{p}}^{2} \mathrm{r}+\dot{\mathrm{u}}_{\mathrm{p}}{ }_{\mathrm{p}} \mathbf{f}\right\}
$$

subject to the observations with the cost functional weightings $Q_{y} \geq 0, r \geq 0$, and $f>0[22]$.

Recently, Davidson and Schmidt introduced a modified optimal control model, or MOCM. The MOCM is easy to implement in current software packages such as MATLAB ${ }^{\text {TM[22]. }}$ As Figure 16 shows, the MOCM retains most of the key 
features of the OCM, except it replaces the OCM's time delay with a $2^{\text {nd }}$ order Pade approximation. The approximation is:

$$
\mathrm{e}^{-\tau \mathrm{s}} \cong \frac{1-\frac{1}{2} \tau \mathrm{s}+\frac{1}{8}(\tau \mathrm{s})^{2}}{1+\frac{1}{2} \tau \mathrm{s}+\frac{1}{8}(\tau \mathrm{s})^{2}}
$$

The Pade' approximation is applied to the pilot's output to reduce system order. The approximation is added to the plant dynamics before the solution of pilot control and estimation gains. Thus, the OCM predictor is not needed by the MOсM. The MOCM also allows one to compute the statespace form of the pilot.

The parameters used by the MOCM for this study are given in Table 4.

Table 4: MOCM Parameters and constants

Input

Effective time delay, $\tau$ (sec)

Neuromotor lag, $\tau_{\mathrm{n}}$ (sec)

Observation noise ratio, $\rho_{\mathrm{y}}$ (dB)

Motor noise ratio, $\rho_{u}(d B)$

objective function input weight, $r$
Value

0.20

0.10

$-20$

$-25$

The objective function input rate weight, $f$, is chosen to produce the desired $\tau_{\mathrm{n}}$.

A Matrix $-X^{T M}$ version of the MOCM algorithm, supplied by J. Davidson of NASA Langley Research Center, was implemented into MATLAB ${ }^{T M}$ for this research. To validate the new implementation, the MOCM was compared to Bacon and Schmidt's 
results obtained with the OCM for a pilot-in-the-loop analysis of a pitch attitude tracking task[24]. The MOCM was compared to Bacon and schmidt's results for Configuration 2D only.

Figure 17 shows the pitch attitude command structure utilized for this comparison. The pilot is located within the dashed box and consists of the combination of a pitch angle loop $(\theta)$ and a pitch angle error loop $\left(\theta_{e}\right)$. The vector of observations $y$ is $\left[\theta_{e} \dot{\theta}_{e} \quad \theta \quad \dot{\theta}\right]$ and the observation weighting matrix $Q_{y}$ is $\operatorname{diag}(16,1,0,0)$, which means $\theta_{e}$ and $\dot{\theta}_{e}$ are weighted. The commanded pitch attitude is driven by a $2^{\text {nd }}$ order white noise disturbance that is characterized by

$$
\ddot{\theta}_{c}+0.25 \dot{\theta}_{c}+0.5 \theta_{c}=0.25 w(t)
$$

The noise, $w(t)$, is a zero mean white noise with intensity of 64. This intensity produces a $4^{\circ} \mathrm{rms}$ value for $\theta_{C}$. The aircraft dynamics for configuration 2D are modeled as

$$
\mathrm{H}_{\mathrm{a}}=\frac{\theta}{\mathrm{F}_{\mathrm{cc}}}=\frac{16.81(0.80 \mathrm{~s}+1)}{\mathrm{s}\left(\mathrm{s}^{2}+6.86 \mathrm{~s}+24.01\right)}
$$

The pilot is able to detect both pitch attitude $(\theta)$ and pitch rate $(\dot{\theta})$. From the pilot state space representation that the MOCM gives, the pilot blocks, or pilot transfer functions, in Figure 17 are computed via the form

$$
\mathrm{H}_{\theta}=\mathrm{h}_{\theta}+\mathrm{sh}_{\dot{\theta}}
$$

where $H_{\theta}$ is the total $\theta$ block, $h_{\theta}$ is the transfer function from $\theta$ to $F_{\text {CC, }}$ and $h_{\dot{\theta}}$ is the transfer function from $\dot{\theta}$ to 


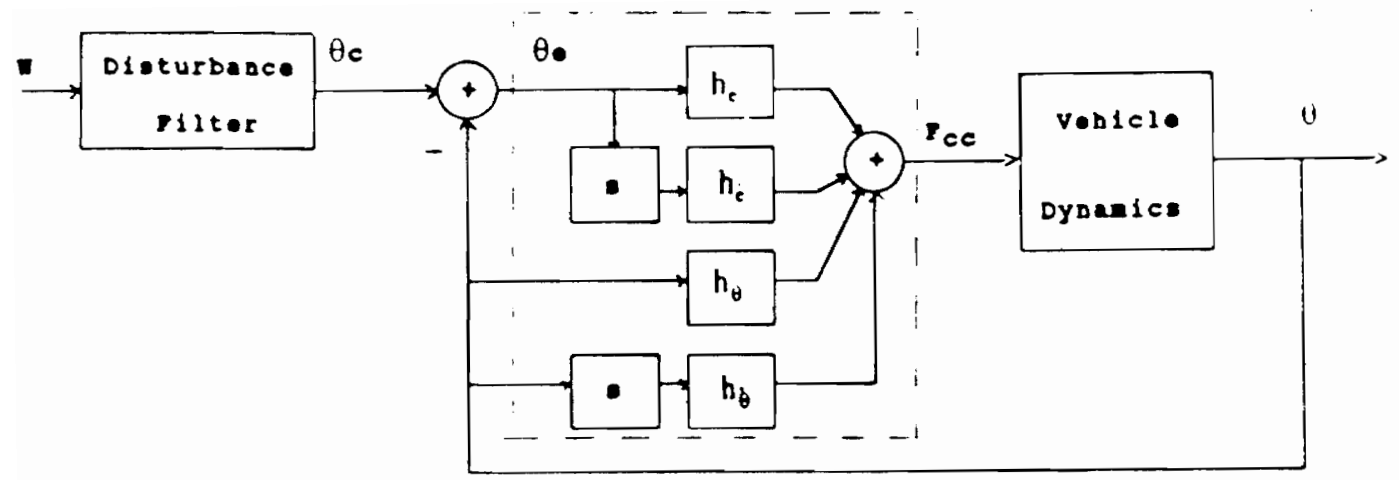

Figure 17: Pitch Attitude Command System 
$F_{C C}$. The blocks for $\theta_{e}$ follow Equation (5-7) to produce $\mathrm{H}_{e}$. Using these blocks, an equivalent, single loop pilot representation is constructed and is given by

$$
H_{p}=\frac{F_{c c}}{\theta_{e}}=\frac{H_{e}}{1-H_{\theta} H_{a}}
$$

Figure 18 gives a comparison of pilot function frequency responses for Bacon and Schmidt's work (OCM) and the MOCM results. The upper plot displays the magnitude of $\mathrm{Hp}$ as a function of frequency whereas the lower plot gives the phase angle of $\mathrm{Hp}$ as a function of frequency. The results matched favorably; however, there are differences that can be attributed to the differences in the two models. For instance, the phase angle does not match at high frequency due to the $2^{\text {nd }}$ order Pade' approximation. A higher order Pade' approximation would match the phase more closely; however the gain would not improve any appreciable amount and the system order would increase.

\subsection{Task Modeling}

The pilot objective during high speed cruise is to maintain a steady altitude (i.e. maintain zero flight path); however, occasionally turbulence disturbs the aircraft and the pilot endeavors to suppress the disturbance in order to maintain the desired cruise altitude. For task modeling, several different pilot loop closure structures were studied. First, as discussed above, Figure 17 shows the 

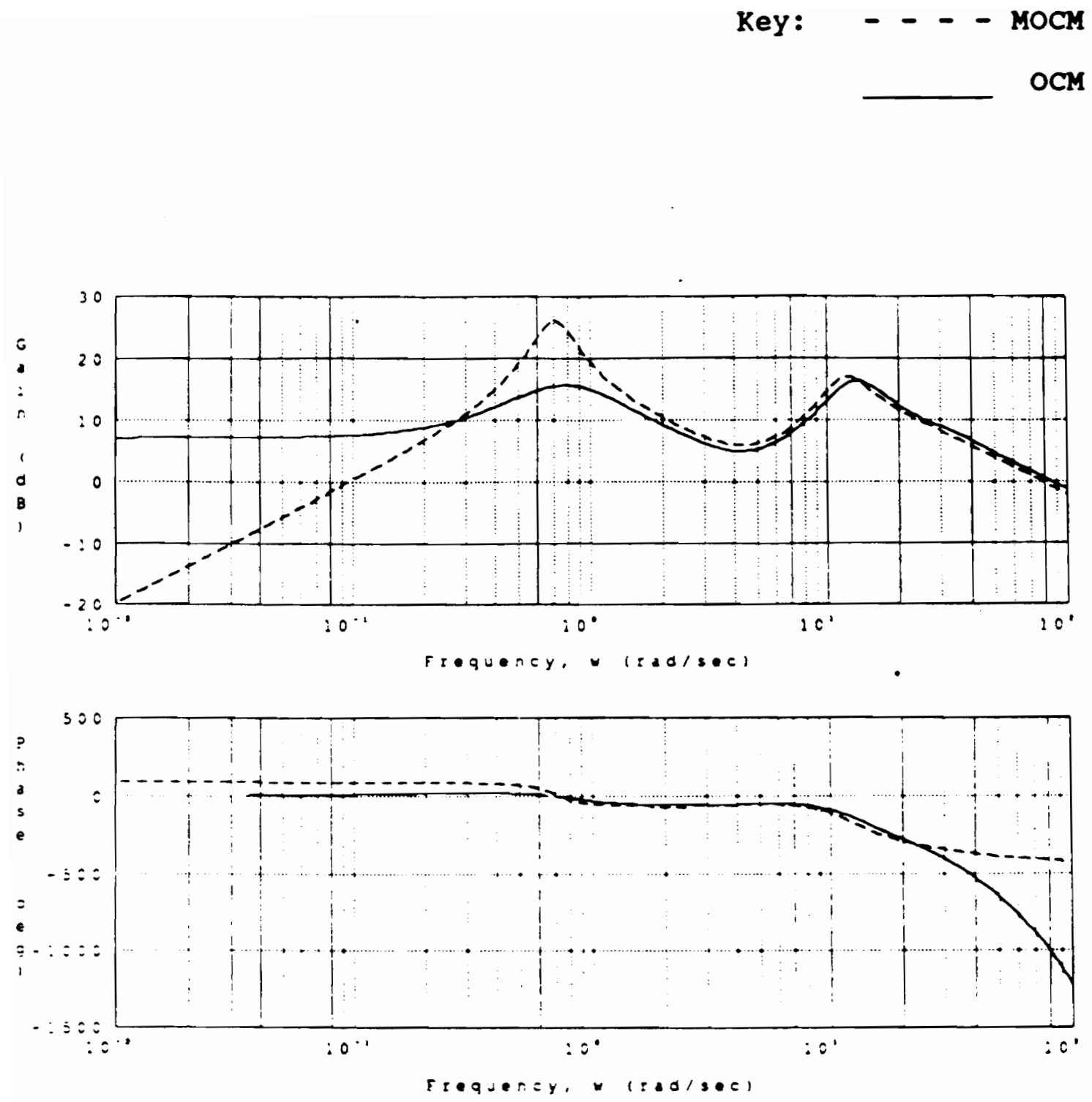

Figure 18: Comparison of Sample MOCM and OCM Results 
pitch attitude command structure utilized in the Bacon and Schmidt analysis. However, as one would expect from the results discussed in chapter 4 , the pitch attitude command structure does not sufficiently represent the constant altitude flight task. Although the attitude loop is essential, an outer loop is required to control sink rate. Thus, the flight path command structure shown in Figure 19 is used for this research. It uses an inner attitude loop and outer flight path loop like that described in Chapter 4. The vector of observations $\mathbf{y}$ is $\left[\begin{array}{llll}\gamma & \dot{\gamma} & \theta & \dot{\theta}\end{array}\right]$ and the observation weighting matrix $Q_{y}$ is $\operatorname{diag}(1,0,0,0)$, which means only flight path angle is weighted.

In addition, this structure is more representative of the actual piloting task because the disturbances are based on the Dryden Turbulence model. The disturbance generates a perturbation in the angle-of-attack. Appendix 2 contains the derivation and associated constants. The result for the turbulence filter, with unity disturbance intensity, is

$$
\frac{\alpha_{\mathrm{g}}(\mathrm{s})}{\mathrm{w}(\mathrm{s})}=\mathrm{f}(\mathrm{s})=\frac{226.12 \mathrm{~s}+180.53}{\mathrm{~s}^{2}+2.7657 \mathrm{~s}+1.9123}
$$

\subsection{Multiloop Pilot Analysis using MOCM}

This section details the integration of the aerodynamic, pilot, and task models in an effort to predict the PIO behavior experienced during the $\mathrm{XB}-70$ flight tests. A correlation between the display characteristics and 


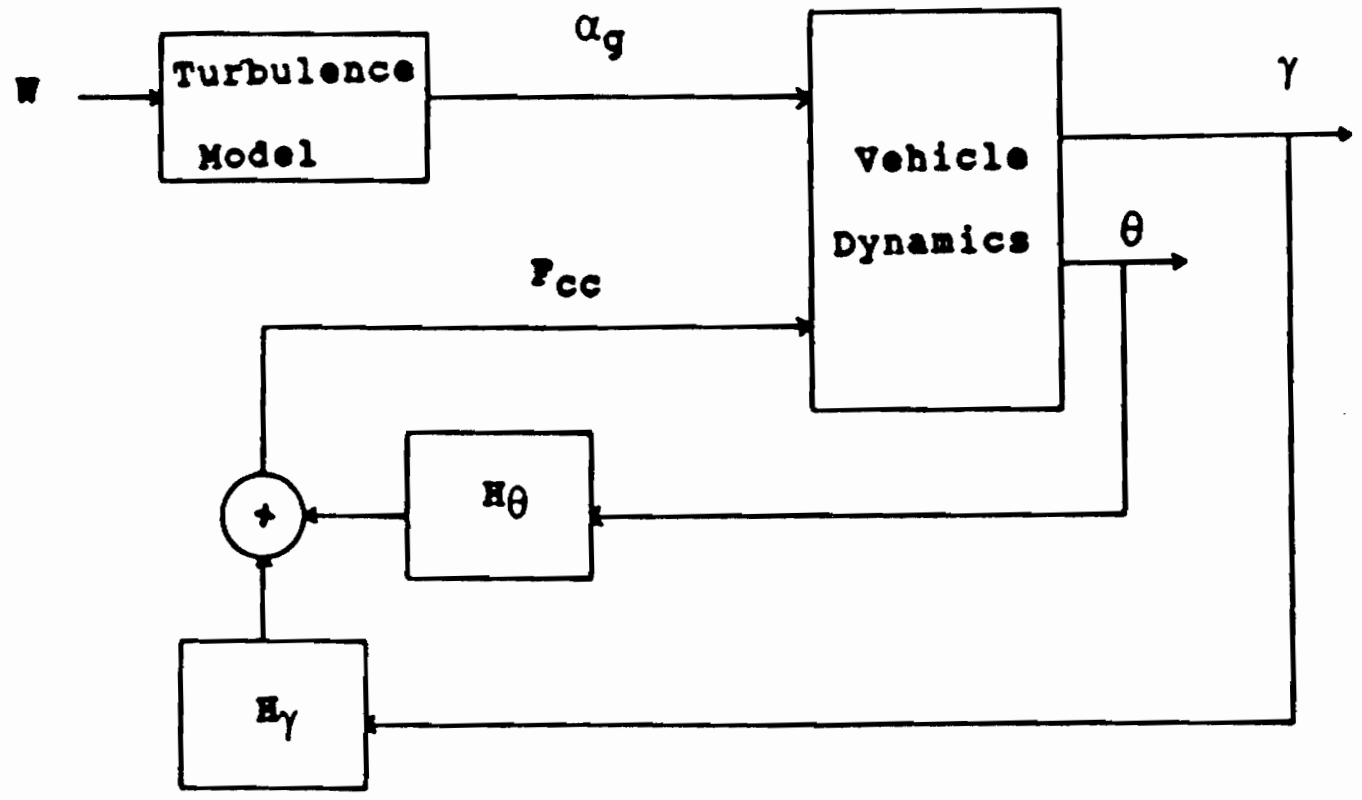

Figure 19: Turbulence Input structure 
altitude excursions or related behavior is sought. The sensor characteristic that will be examined is the attitude displays' resolution as modeled by pilot threshold on the attitude input. Decreasing resolution of the attitude display is represented in the modeling process by increasing attitude threshold. From Equations (5-1) and (5-2), one can see that as the pitch threshold $\mathrm{a}_{\mathrm{TH}_{i}}$ increases, $\operatorname{erf}()$ approaches unity. The noise in the pitch signal therefore increases to mimic the effect of lost signal information.

The expected results are increased root-mean-square (rms) signals in flight path and altitude when the pitch attitude threshold is increased. Increased rms values mean that the pilot has difficulty in maintaining constant altitude flight. Furthermore, it is expected that the gain of the $\mathrm{H}_{\theta}$ block will decrease with increasing threshold. This result is expected because the pilot will use pitch information less and will finally ignore the attitude information altogether.

\subsubsection{Methodology}

The MOсM algorithm is first implemented with all observation thresholds set to zero. Then, while the thresholds on flight path and flight path rate are fixed at zero, the thresholds on pitch angle and pitch-rate are varied according to Equation $(5-10)$.

$$
\left[\theta_{\mathrm{TH}} \dot{\theta}_{\mathrm{TH}}\right]^{\mathrm{T}}=\left[\theta_{\mathrm{RMS}} \dot{\theta}_{\mathrm{RMS}}\right]^{\mathrm{T}} *_{2} \mathrm{~N}
$$


In Equation (5-10), the subscript $\mathrm{TH}$ represents threshold value and the subscript RMS represents the rms value at zero thresholds. The integer value of $\mathbf{N}$ is varied to produce a range of thresholds. The pitch rate threshold is varied with attitude threshold because it is assumed that a pilot can deduce pitch rate information from the pitch attitude display.

\subsubsection{Multiloop Pilot Discussion}

Once the MOCM algorithm is executed with all thresholds set to zero, the $\mathrm{H}_{\gamma}$ and $\mathrm{H}_{\theta}$ blocks are computed to determine the closure scheme implemented by the MOCM. The frequency responses of $\mathrm{H}_{\theta}$ and $\mathrm{H}_{\gamma}$ are given in Figures 20 and 21, respectively. Note that at low frequencies the phase angle is $180^{\circ}$ for $\mathrm{H}_{\theta}$ and zero degrees for $\mathrm{H}_{\gamma}$. The MOCM algorithm utilizes the following equation for the computation of the pilot output.

$$
\mathrm{F}_{\mathrm{CC}}=\mathrm{H}_{\theta} \theta+\mathrm{H}_{\gamma} \gamma
$$

This equation can be rearranged to look like the characteristic equations used in Chapter 4.

$$
1-\mathrm{H}_{\theta}\left(\theta / \mathrm{F}_{\mathrm{CC}}\right)-\mathrm{H}_{\gamma}\left(\gamma / \mathrm{F}_{\mathrm{CC}}\right)=0
$$

In Chapter 4, the gains were considered greater than or equal to zero. From the frequency responses given in Figures 20 and 21, the phase angle plot gives the sign of the transfer function. Since $\mathrm{H}_{\gamma}$ begins at zero degrees, its sign is considered greater than zero; however, $\mathrm{H}_{\theta}$ begins at 

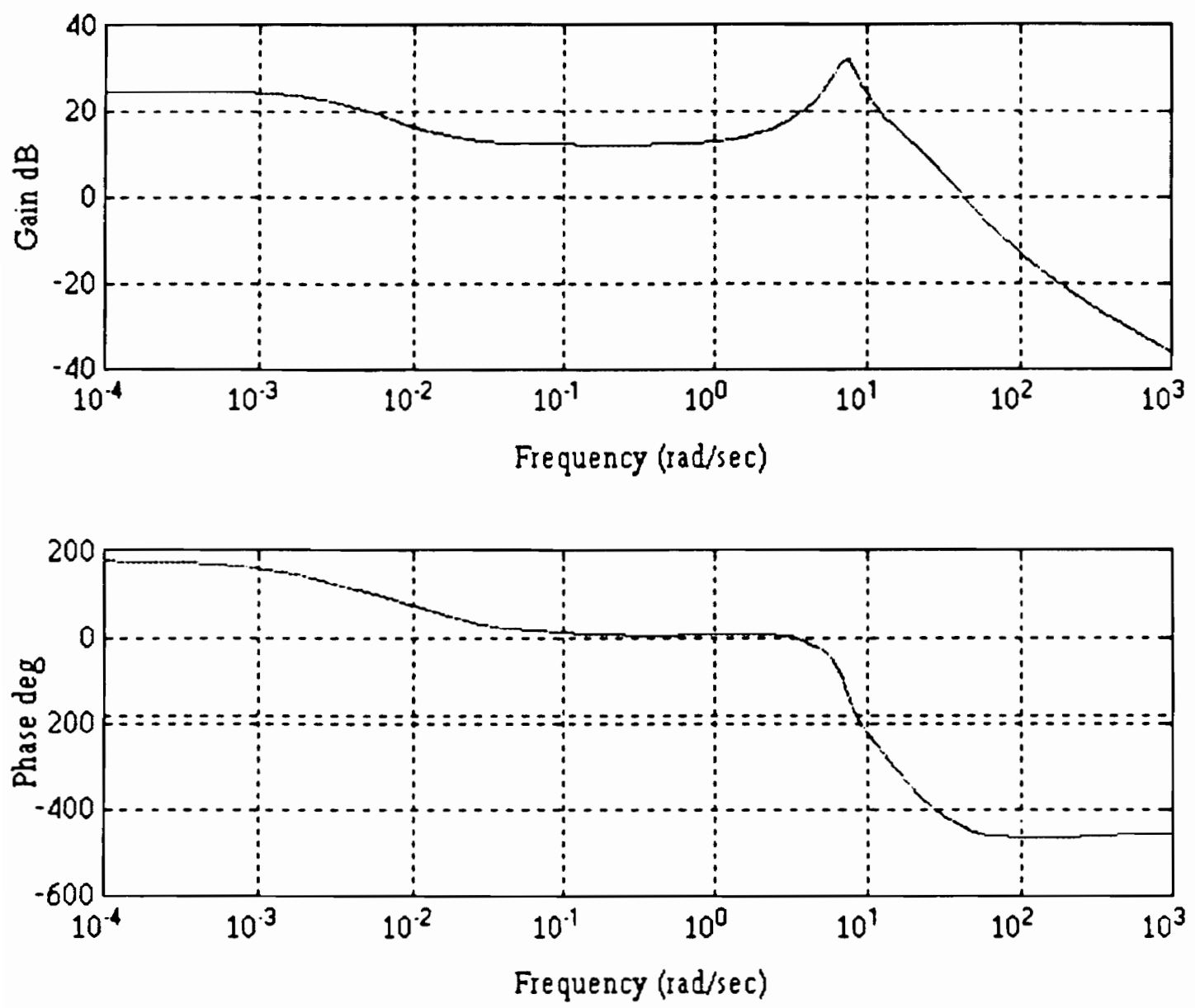

Figure 20: Pilot Frequency Response to Pitch Attitude 


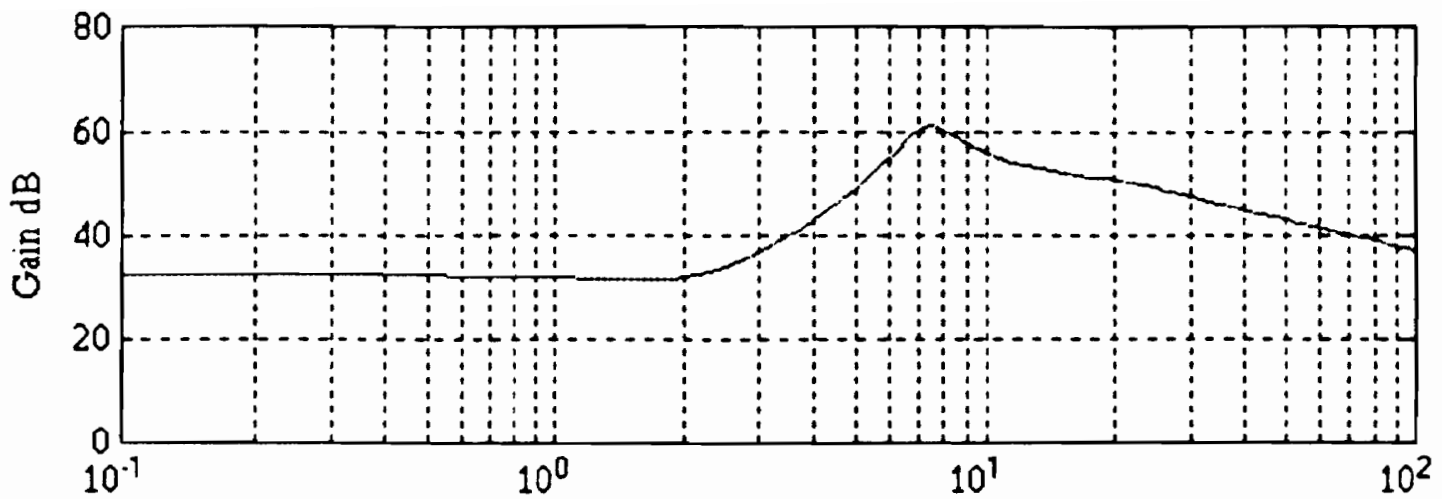

Frequency (rad/sec)

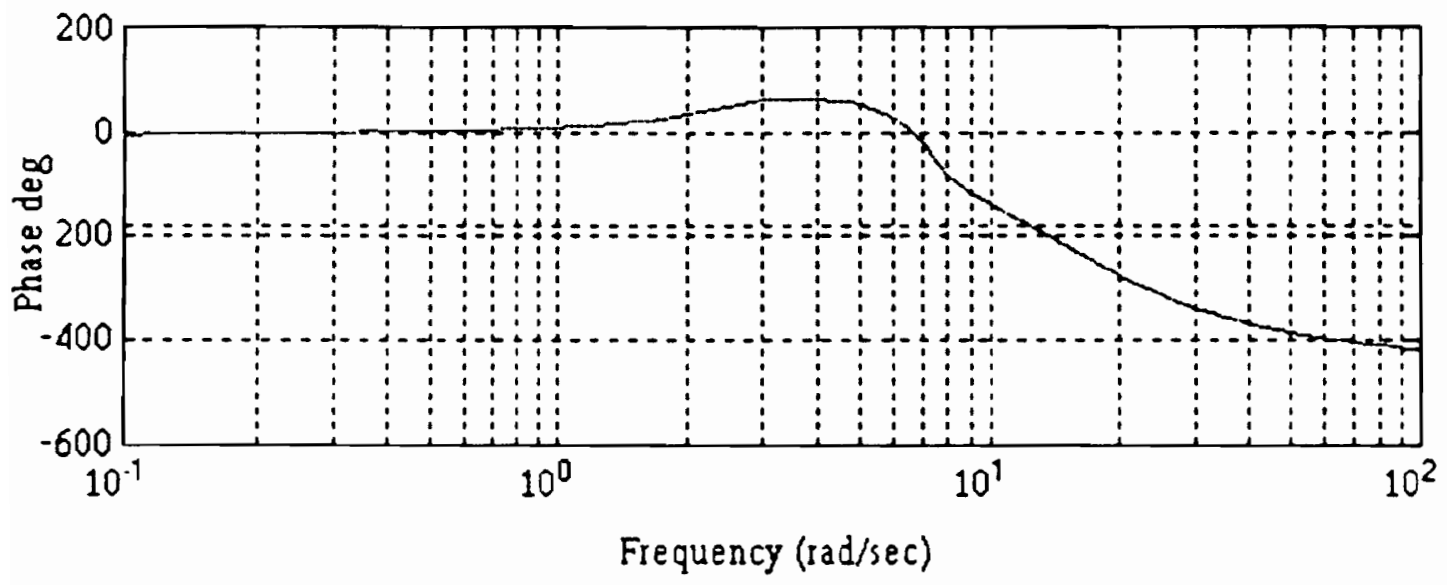

Figure 21: Pilot Frequency Response to Flight Path 
$180^{\circ}$ and its sign is considered negative. With these facts one can see that the MOcM pilot control structure is the same structure suggested by the sequential loop closure analysis performed in Chapter 4.

Figures 22 and 23 show the effects of increased thresholds on the rms values of $\gamma$ and $h$, respectively. In Figures 22-a and 23-a, the value of pitch angle threshold is plotted along the abscissa and the signal rms value is on the ordinate. In Figures $22-b$ and $23-b$, the abscissa is the integer value of $\mathbf{N}$ and on the ordinates are the rms values.

The results show that as the thresholds on pitch and pitch-rate increase the rms values of flight path and altitude increase. As the thresholds increase, the rms values begin to increase also, then the rms values rapidly increase, and finally level off. Thus, the altitude excursions are predicted using modern control methods. Additional rms data for other signals are presented in Appendix 3 .

The most significant increase in the rms values occurs in the attitude threshold range of $1.09^{\circ}$ to $4.34^{\circ}$, or the $\mathrm{N}$ range of -1 to 1 . From this result, one can conclude that if the pitch attitude display resolution is greater than $1^{\circ}$ performance will begin to degrade. For a display resolution greater than $4^{\circ}$, performance is severely degraded and altitude excursions are of high probability. For HSCT 


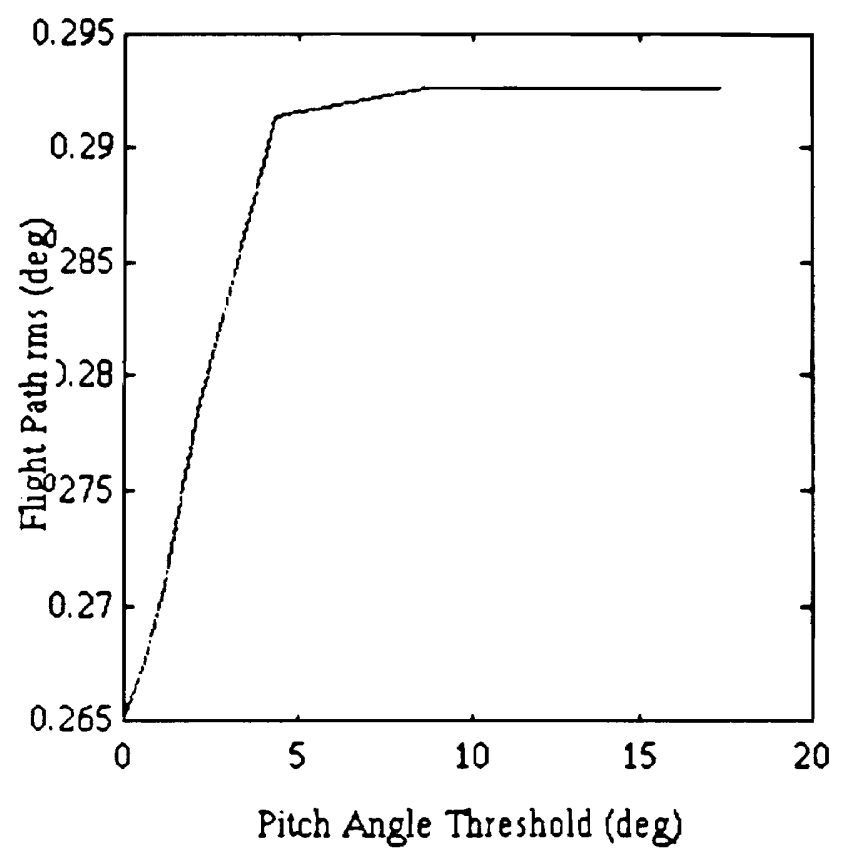

Figure 22-a: Flight Path rms versus Attitude Threshold

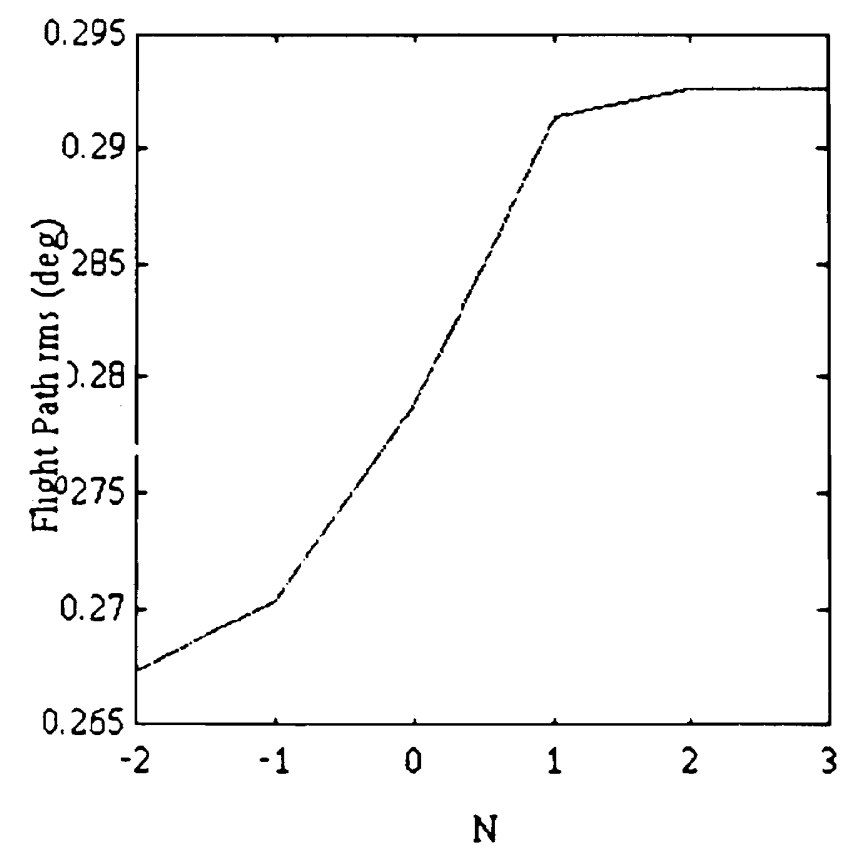

Figure 22-b: Flight Path rms versus $\mathrm{N}$ 


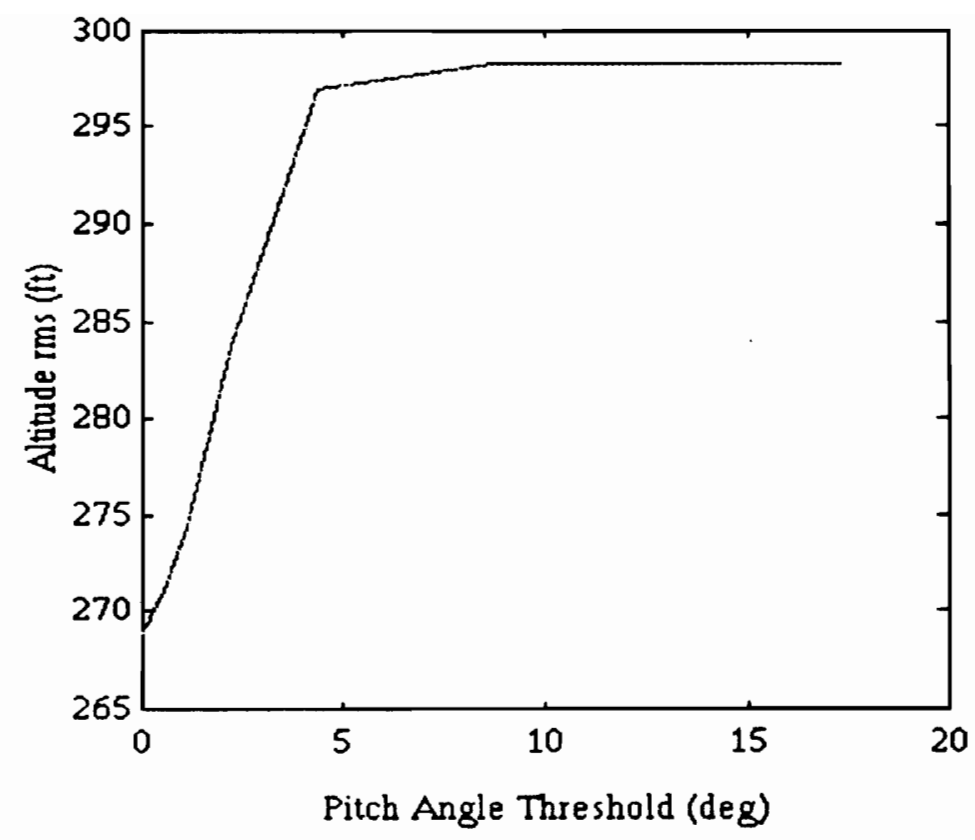

Figure 23-a: Altitude rms versus Attitude Threshold

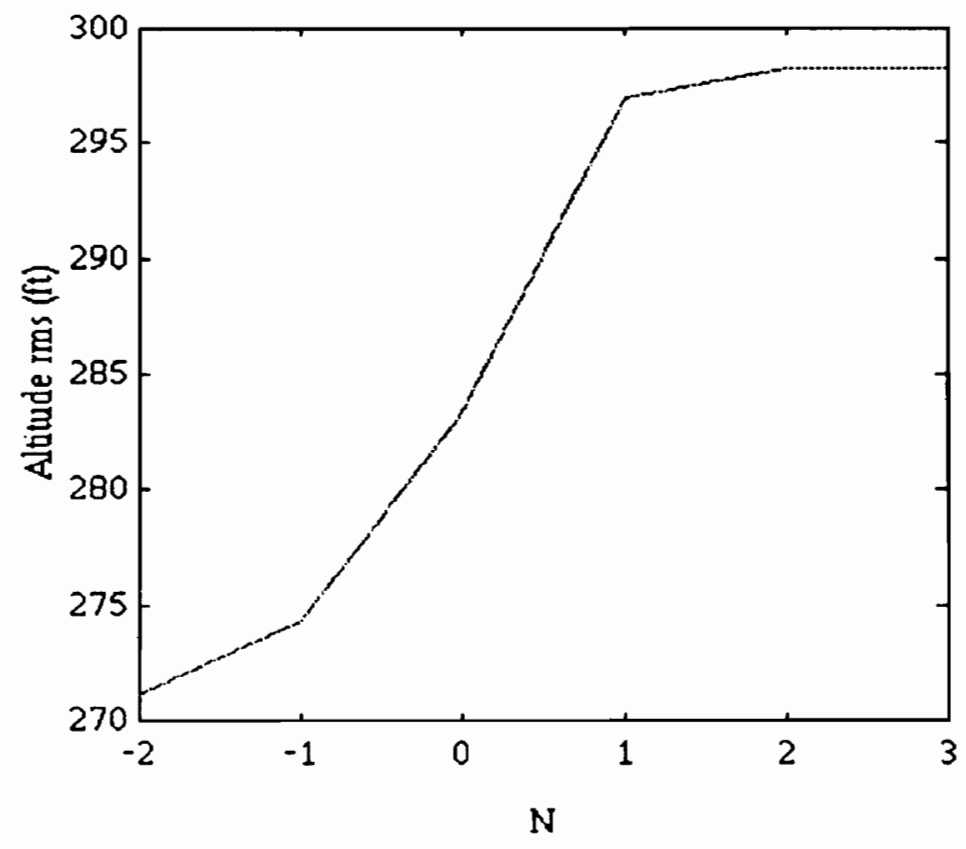

Figure 23-b: Altitude rms versus $\mathrm{N}$ 
aircraft, tight altitude control is required for passenger comfort, thus the critical value for pitch attitude display resolution is at most $1^{\circ}$.

The increase in threshold is modeled as an increase in white observation noise (Equation (5-1)). As the noise increases, the signal becomes less reliable. The leveling off of the rms values represent the pilot totally ignoring the attitude information due to the overwhelming observation noise. Without the attitude information, the inner loop essentially vanishes. This conjecture is proven in Figure 24 which shows the frequency responses of $\mathrm{H}_{\theta}$ parameterized by $N$. As $N$ increases, one can readily see that the magnitude of $\mathrm{H}_{\theta}$ decreases. For $\mathrm{N}=3$ (not shown) the magnitude of $\mathrm{H}_{\theta}$ is practically zero. Once the pilot doubts the attitude information, the gain of $\mathrm{H}_{\theta}$ decreases and the phugoid mode damping degrades. Finally, for $\mathrm{N}=2$ or 3 , the pilot does not effectively have an inner attitude loop. As detailed in Chapter 4, the phugoid mode destabilizes quickly for flight path loop closure without pitch closure. 


$\begin{array}{lll}\text { Key: } & * & \text { Zero Thresholds } \\ -2 & N=-2 \\ -1 & N=-1 \\ 0 & N=0 \\ 1 & N=1 \\ 2 & N=2\end{array}$
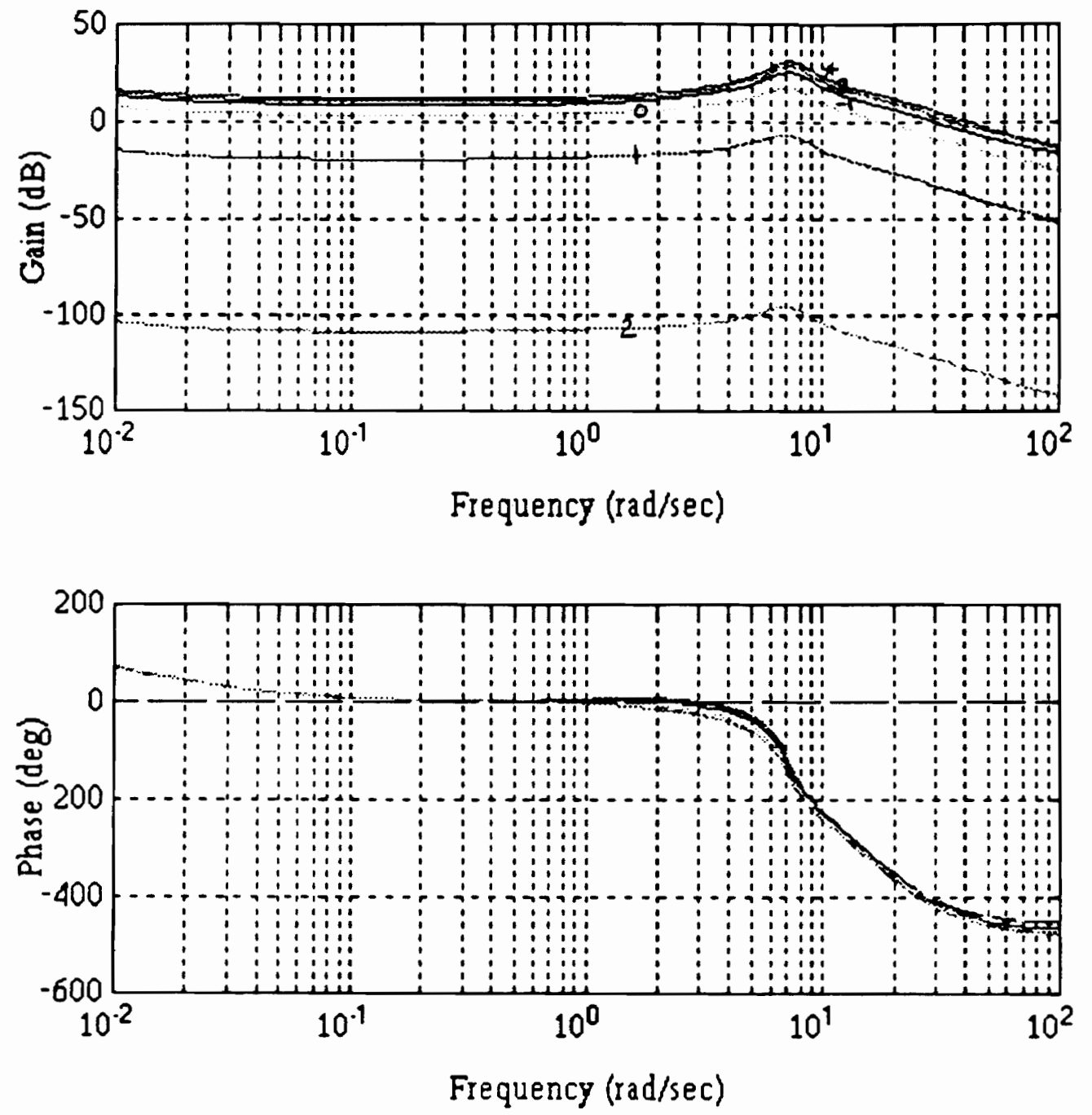

Figure 24: Ho variation with Attitude Threshold 


\subsection{Conclusion}

Modern and classical control theories have been applied to a representative supersonic transport in order to predict pilot induced altitude excursions. This research has successfully predicted the phenomenon with a model of the XB-70 aircraft. Specific results show that:

1. The critical value of pilot attitude threshold is roughly $1^{\circ}$. This figure can be interpreted as the display characteristic necessary for acceptable aircraft performance.

2. For pitch attitude thresholds greater than $4^{\circ}$, pilot/aircraft performance is severely degraded because the pilot will undoubtedly ignore the attitude indicator.

3. An aircraft with otherwise good handling qualities can exhibit poor performance or undesirable behavior if the cockpit displays are corrupted or 'noisy'.

4. Acceptable performance can be achieved despite noisy displays if the noise does not prohibit the pilot from executing critical loop closures.

5. Modern control theory and classical root-locus methods arrive at the same conclusions concerning longitudinal pilot-vehicle interactions for high speed cruising aircraft such as the $\mathrm{XB}-70$ or HSCT.

Future work would expand the analysis to include sensor lags and rate of climb sensors. Also, it would be of 
interest to repeat this research for other tasks and flight conditions. 


\subsection{References}

1. Powers, Bruce G. A Review of Transport Handling Qualities Criteria in Terms of Preliminary XB-70 Flight Experience. Flight Research Center, Edwards, CA. May, 1968. NASA TM X-1584.

2. Wolowicz, Chester, and Yancey, Roxanah. Summary of stability and Control Characteristics of XB-70 Airplane. Flight Research Center, Edwards, CA. October, 1973. NASA TM X-2933.

3. Wykes, John and Lawerence, Robert. Estimated Performance and stability Control Data for Correlation with $\mathrm{XB}-70-1$ Test Data. North American Rockwell Corporation, Los Angeles, CA. July, 1971. NASA CR 114335.

4. Wolowicz, Chester, Strutz, Larry, Gilyard, Glenn, and Matheny, Neil w. Preliminary Flight Evaluation of the stability and Control Derivatives and Dynamic Characteristics of the Unaugmented XB-70-1 Airplane including comparisons with Predictions. Flight Research Center, Edwards, CA. May, 1968. NASA TN D-4578.

5. Wolowicz, Chester, and Yancey, Roxanah. Comparison of Predictions of the $\mathrm{xB}-70-1$ Longitudinal stability and Control Derivatives with Flight Results for Six Flight Conditions. Flight Research center, Edwards, CA. August, 1970. NASA TM X-1881.

6. Jenkins, Jerald, DeAngelis, V. Michael, Friend, Edward, and Monaghan, Richard. Flight Measurements of Canard Loads, Canard Buffeting, and Elevon and Wing-Tip Hinge Moments on XB-70 Aircraft including Comparisons with Predictions. Flight Research Center, Edwards, CA. August, 1969. NASA TND-5359.

7. Gallagher, Robert. Investigation of a Digital Simulation of the XB-70 Inlet and its Application to Flight-Experienced Free-stream Disturbances at Mach numbers of 2.4 to 2.6. Flight Research Center, Edwards, CA. June, 1970. NASA TN D-5827. 
8. Martin, Arnold W., and Beaulieu, warren D. $\underline{X B-70}$ Flight Test Data Comparisons with simulation Predictions of Inlet Unstart and Buzz. North American Rockwell Corporation, Los Angeles, $C A$. June, 1970. NASA CR-1631.

9. Stenton, Thomas E. Theoretical Frequency Response Functions and Power spectra of the XB-70 Response to Atmospheric Turbulence. North American Rockwell Corporation, Los Angeles, CA. August, 1970. NASA CR-1621.

10. Saltzman, Edwin J., Goecke, Sheryll, and Rembo, Chris. Base Pressure Measurements on the XB-70 Airplane at Mach Numbers from 0.4 to 3.0. Flight Research Center, Los Angeles, CA. September, 1968. NASA TM X-1612.

11. Wolowicz, Chester. Analysis of an Emergency Deceleration and Decent of the XB-70-1 Airplane due to Engine Damage Resulting from structural Failure. Flight Research Center, Edwards, CA. March, 1966. NASA TM X-1195.

12. Arnaiz, Henry H. Flight Measured Lift and Drag Characteristics of a Large, Flexible, High Supersonic cruise Airplane. Dryden Flight Research Center, Edwards, CA. May, 1977. NASA TM $\mathrm{X}-3532$.

13. Andrews, William H. Summary of Preliminary Data Derived from the XB-70 Airplanes. Flight Research Center, Edwards, CA. June, 1966. NASA TM X-1240.

14. Daugherty, James $c$. wind-Tunnel/Flight Correlation study of Aerodynamic Characteristics of a Large Flexible Supersonic Cruise Airplane (XB-70-1) Part I. Ames Research Center, Moffett Field, CA. November, 1979. NASA TP 1514.

15. Peterson, John, Mann, Michael, Sorrells, Russell B., Sawyer, Wallace C., and Fuller, Dennis. WindTunnel/Flight correlation study of Aerodynamic Characteristics of a Large Flexible supersonic Cruise Airplane (XB-70-1) Part II. Langley Research Center, Hampton, VA. Feb., 1980. NASA TP-1515. 
16. Arnaiz, Henry H., Peterson, John B., and Daugherty, James C. Wind-Tunnel/Flight Correlation study of Aerodynamic Characteristics of a Large Flexible Supersonic Cruise Airplane (XB-70-1) Part III. Langley Research Center, Hampton, VA. March, 1980. NASA TP-1516.

17. McKay, James M., Kordes, Eldon E., and Wykes, John H. Flight Investigation of $\mathrm{XB}-70$ structural Response to Oscillatory Shaker Excitation and Correlation with Analytical Results. Flight Research Center, Edwards, CA. April, 1973. NASA TN D-7227.

18. Heffley, R. K., et al. Aircraft Handling Qualities Data. System Technology, Inc. Dec., 1972. NASA CR-2144.

19. Military standard: Flying oualities of Piloted Aircraft. MIL-STD-1797. 31 March 1987.

20. Craig, Samuel J., and Heffley, Robert K. Factors Governing control in a STOL Landing Approach. Journal of Aircraft, August, 1973. Vol. 10, Number 8, pp. 495-502.

21. Kleinman, D. L., Baron, S., and Levison, W. H. An optimal Control Model of Human Response Part I: Theory and Validation. Automatica, 1970, Vol. 6, pp. 357-369.

22. Davidson, John B., and Schmidt, David K. Modified optimal Control Pilot Model for Computer-Aided Design and Analysis. Langley Research Center, Hampton, VA. October, 1992. NASA TM-4384.

23. Graham, Dunstan and McRuer, Duane. Analysis of Nonlinear Control systems. John wiley \& Sons, Inc. New York, NY. 1961. p. 236.

24. Bacon, B. J., and Schmidt, David K. A Modern Approach to Pilot/Vehicle Analysis and the NealSmith Criteria. 1982 AIAA Atmospheric Flight Mechanics Conference, San Diego, CA. AIAA Paper 82-1357.

25. Stevens, Brain L., and Lewis, Frank L. Aircraft Control and Simulation. John Wiley \& Sons, Inc. New York, NY. 1992, pp. 101-102.

26. Friedland, Bernard. Control system Design. McGrawHill, st. Louis, MO. 1986. Section 10.6. 


\subsection{Appendix 1: State space Models of the XB-70}

From References 1-18, a database for the XB-70 was created and implemented in MATLAB $^{T M}$. From the database, a longitudinal model was extracted for research[25]. The cruise flight condition statistics are given in Table 5 .

Table 5: Cruise Flight Condition

$\begin{array}{ll}\left.I_{y} \text { (slug-ft }{ }^{2}\right) & 10^{6} \\ \text { c (ft) } & 78.53 \\ \text { S (ft ) (lbs.) } & 6,297.8 \\ \text { Weight (lt) } & 384,524 \\ \text { Altitude (ft) } & 60,000 \\ \text { Velocity (ft/sec) } & 2,420 \\ \text { Mach Number } & 2.5 \\ \text { Trim angle-of-attack }\left({ }^{\circ}\right) & 3.4 \\ \text { Trim elevator deflection }\left({ }^{\circ}\right) & 2.7\end{array}$

For the $\mathrm{xB}-70^{\prime} \mathrm{s}$ Iinear system representation, $\dot{\mathrm{x}}=\mathrm{ax}+\mathrm{bu}$, the state vector $x$ is $\left[v^{\prime} \alpha q \theta\right]^{T}$ and the control vector $\mathrm{u}$ is $\left[\begin{array}{llll}\delta_{t} & \delta_{e} & \delta_{c}\end{array}\right]^{\mathrm{T}}$. The a and $\mathrm{b}$ matrices are:

$$
\begin{aligned}
& \mathbf{a}=\left[\begin{array}{rrrr}
-3.0648 e-3 & 8.5233 e+0 & 0 & -3.2170 e+1 \\
-5.5914 e-6 & -2.1590 e-1 & 1.0000 e+0 & 0 \\
7.0583 e-5 & -2.9055 e+0 & -2.7577 e-1 & 0 \\
0 & 0 & 1.0000 e+0 & 0
\end{array}\right] \\
& b=\left[\begin{array}{rrr}
8.3622 e-5 & 1.0710 e+0 & 1.3619 e+0 \\
0 & -7.3756 e-3 & -9.3790 e-3 \\
2.2000 e-7 & -1.0518 e+0 & 2.7679 e+0 \\
0 & 0 & 0
\end{array}\right]
\end{aligned}
$$

The linear system representation of the bare airframe and PACS is denoted $\dot{\mathrm{x}}=\mathrm{Ax}+\mathrm{Bu}$. The linear system variables $\mathrm{x}$ and $\mathrm{u}$ are $\left[\mathrm{v}^{\prime} \alpha\right.$ q $\theta \delta_{\mathrm{e}} \sim \delta_{\mathrm{e}}^{*} \delta_{\mathrm{cc}} \sim \frac{\mathrm{d}}{\mathrm{dt}}\left(\delta_{\mathrm{Cc}} \sim \delta_{\mathrm{cc}}^{*} \frac{\mathrm{d}}{\mathrm{dt}}\left(\delta_{\mathrm{Cc}}{ }^{*}\right)\right]^{\mathrm{T}}$ and $\left[\mathrm{F}_{\mathrm{CC}} \delta_{t}\right]^{\mathrm{T}}$, respectively. The $\mathrm{A}$ and $\mathrm{B}$ matrices are given below for the PACS's SAS on and off. 
First, for the cruise flight condition with the SAS on: $\mathrm{A}=$ Columns 1 through 6

$\left\{\begin{array}{rrrrrr}-3.065 e-3 & 8.523 e+0 & 0 & -3.217 e+1 & 1.071 e+0 & 1.071 e+0 \\ -5.591 e-6 & -2.159 e-1 & 1.000 e+0 & 0 & -7.376 e-3 & -7.376 e-3 \\ 7.058 e-5 & -2.906 e+0 & -2.758 e-1 & 0 & -1.052 e+0 & -1.052 e+0 \\ 0 & 0 & 1.000 e+0 & 0 & 0 & 0 \\ -1.837 e-2 & 1.319 e+1 & 2.566 e+1 & 6.284 e+0 & -1.420 e+1 & -8.706 e-1 \\ 0 & 0 & 0 & 0 & 0 & -1.333 e+1 \\ 0 & 0 & 0 & 0 & 0 & 0 \\ 1.492 e-1 & 7.280 e+3 & 7.437 e+1 & 0 & 5.056 e+2 & 5.056 e+2 \\ 0 & 0 & 0 & 0 & 0 & 0 \\ 0 & 0 & 0 & 0 & 0 & 0\end{array}\right.$

Columns 7 through 10

$\left.\begin{array}{rrrr}1.664 e-2 & 0 & 1.664 e-2 & 0 \\ -1.146 e-4 & 0 & -1.146 e-4 & 0 \\ 3.382 e-2 & 0 & 3.382 e-2 & 0 \\ 0 & 0 & 0 & 0 \\ -1.913 e+0 & 0 & -8.312 e-1 & 0 \\ 0 & 0 & -1.082 e+0 & 0 \\ 0 & 1.000 e+0 & 0 & 0 \\ -1.006 e+3 & -1.400 e+1 & -5.672 e+0 & 0 \\ 0 & 0 & 0 & 1.000 e+0 \\ 0 & 0 & -1.000 e+3 & -1.400 e+1\end{array}\right]$

$\mathrm{B}=$

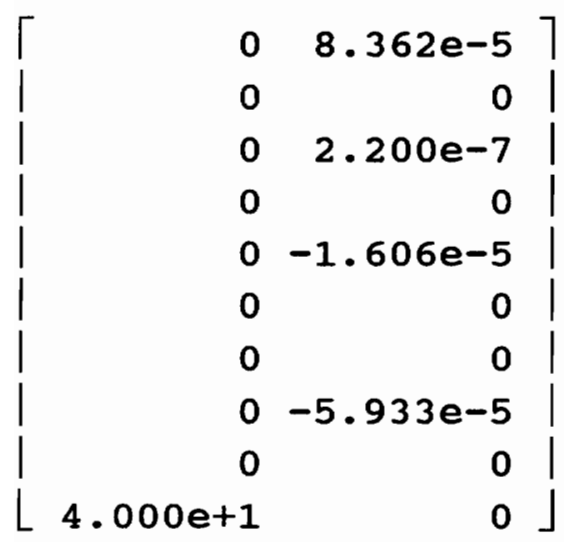


Second, the cruise flight condition with the SAS off:
$\mathrm{A}=$
Columns 1 through 6
$\left\{\begin{array}{rrrrrr}-3.065 e-3 & 8.523 e+0 & 0 & -3.217 e+1 & 1.071 e+0 & 1.071 e+0 \\ -5.591 e-6 & -2.159 e-1 & 1.000 e+0 & 0 & -7.376 e-3 & -7.376 e-3 \\ 7.058 e-5 & -2.906 e+0 & -2.758 e-1 & 0 & -1.052 e+0 & -1.052 e+0 \\ 0 & 0 & 1.000 e+0 & 0 & 0 & 0 \\ 0 & 0 & 0 & 0 & -1.333 e+1 & 0 \\ 0 & 0 & 0 & 0 & 0 & -1.333 e+1 \\ 0 & 0 & 0 & 0 & 0 & 0 \\ 1.492 e-1 & 7.280 e+3 & 7.437 e+1 & 0 & 5.056 e+2 & 5.056 e+2 \\ 0 & 0 & 0 & 0 & 0 & 0 \\ 0 & 0 & 0 & 0 & 0 & 0\end{array}\right.$

Columns 7 through 10

$\left.\begin{array}{rrrr}1.664 \mathrm{e}-2 & 0 & 1.664 \mathrm{e}-2 & 0 \\ -1.146 \mathrm{e}-4 & 0 & -1.146 \mathrm{e}-4 & 0 \\ 3.382 \mathrm{e}-2 & 0 & 3.382 \mathrm{e}-2 & 0 \\ 0 & 0 & 0 & 0 \\ -1.082 \mathrm{e}+0 & 0 & 0 & 0 \\ 0 & 0 & -1.082 \mathrm{e}+0 & 0 \\ 0 & 1.000 \mathrm{e}+0 & 0 & 0 \\ -1.006 \mathrm{e}+3 & -1.400 \mathrm{e}+1 & -5.672 \mathrm{e}+0 & 0 \\ 0 & 0 & 0 & 1.000 \mathrm{e}+0 \\ 0 & 0 & -1.000 \mathrm{e}+3 & -1.400 \mathrm{e}+1\end{array}\right]$

$\mathrm{B}=$

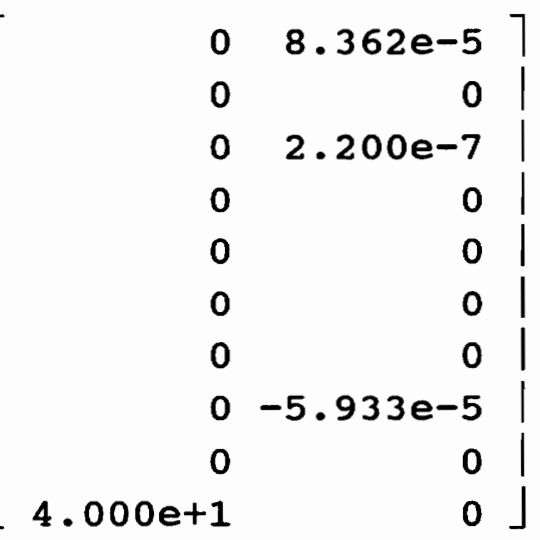




\subsection{Appendix 2: Turbulence Model}

The Dryden model is utilized for turbulence disturbances. From Appendix A of MIL-STD-1797[19], the power spectra of the plunge turbulence is

$$
\Phi_{\mathrm{wg}}(\Omega)=\sigma^{2} \frac{2 L_{\mathrm{w}}}{\pi}\left[\frac{1+12\left(\mathrm{~L}_{\mathrm{w}} \Omega\right)^{2}}{\left(1+4\left(\mathrm{~L}_{\mathrm{w}} \Omega\right)\right)^{2}}\right]
$$

To develop a turbulence filter for pilot-vehicle study, Equation (9-1) was factored to yield Equation (9-2).

$$
\Phi_{\text {wg }}(s)=\sigma^{2} \frac{2 L_{w}}{\pi}\left[\frac{\left(1+\sqrt{12} \frac{L_{w}}{V} s\right)\left(1-\sqrt{12} \frac{L_{w}}{V} s\right)}{\left(1+2 \frac{L_{w}}{V} s\right)^{2}\left(1-2 \frac{L_{w}}{V} s\right)^{2}}\right]
$$

The causal and non-causal components were then separated.

$$
\Phi_{\mathrm{wg}}(\mathrm{s})=\mathrm{f}(\mathrm{s}) * \mathrm{f}(-\mathrm{s})
$$

Comparing Equations (9-2) and (9-3), the filter for the turbulence model is extracted (9-4).

$$
f(s)=\sigma \sqrt{\frac{3 V^{2}}{2 \pi L_{w}}}\left[\frac{\left(s+\frac{V}{\sqrt{12} L_{w}}\right)}{\left(s+\frac{V}{2 L_{w}}\right)^{2}}\right]
$$

From Reference 19, the parameters are chosen to correspond to "moderate" turbulence at 60,000 ft. Thus, $\sigma$ is set to $4 \mathrm{ft} / \mathrm{sec}$, and the value for $L_{w}$ is $875 \mathrm{ft}$. $v$, the trim velocity, is $2,420 \mathrm{ft} / \mathrm{sec}$ for the cruise flight condition. Filling in Equation (9-4) with the constants, the turbulence filter becomes: 


$$
f(s)=\frac{226.12 s+180.53}{s^{2}+2.7657 s+1.9123}
$$

The filter in Equation $(9-5)$ is used as the turbulence model in the block diagram of Figure 19.

The following rms definition is utilized in this research[26]:

$$
\sigma_{w}^{2}=\frac{1}{2 \pi} \int_{-\infty}^{\infty} \Phi_{w g}(\omega) \mathrm{d} \omega
$$

The parameters of the chosen turbulence filter lead to a value of $111 \mathrm{ft} / \mathrm{sec}$ for $\sigma_{w}$ which translates into an angle of attack disturbance rms of about $3^{\circ}$. 


\subsection{Appendix 3: RMs Plots}

For reference, Appendix 3 contains additional rms data of pilot input and output signals. Figure 25 shows the how the flight path rate rms values vary with attitude threshold. Next, Figure 26 gives the rms plots for pitch attitude. Then Figure 27 displays the relationship between pitch rate rms and varying attitude threshold. Finally, Figure 28 exhibits the variation of pilot stick force rms with attitude threshold. 


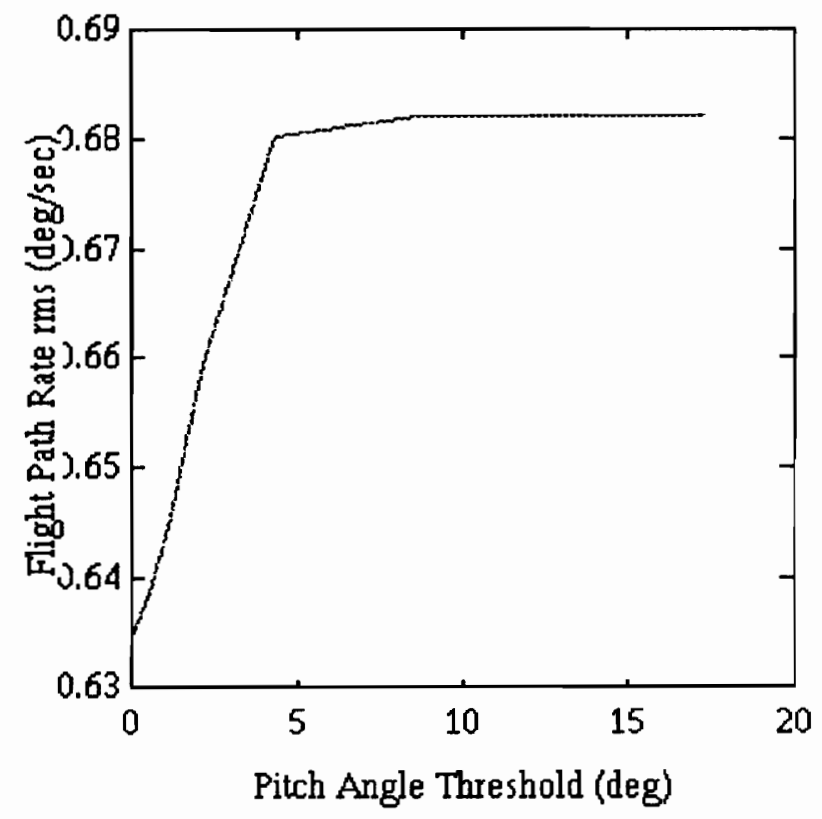

Figure 25-a: Flight Path Rate rms vs. Attitude Threshold

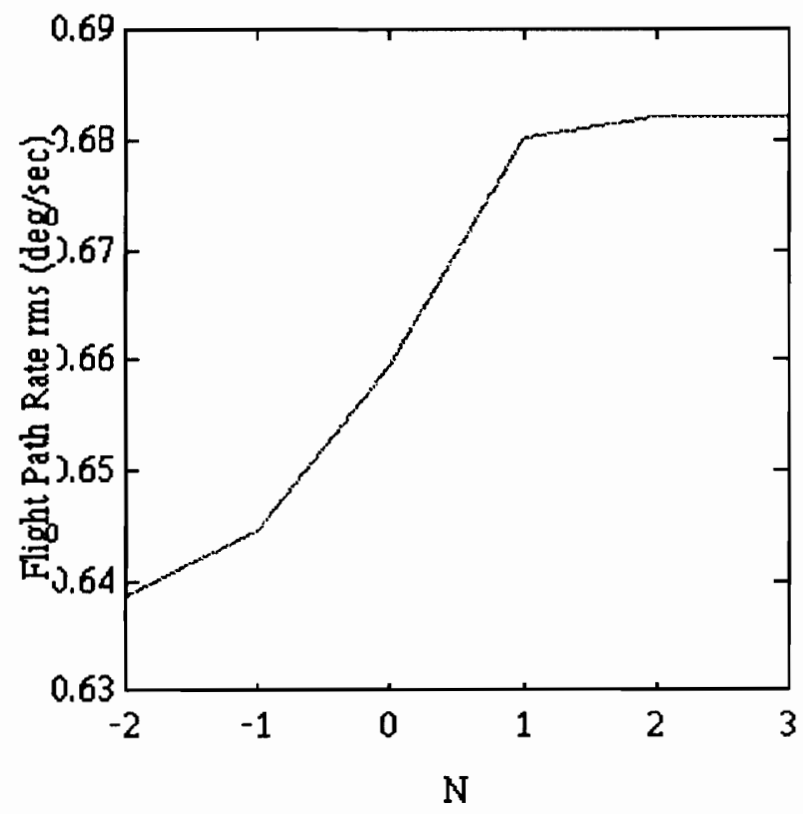

Figure 25-b: Flight Path Rate rms vs. N 


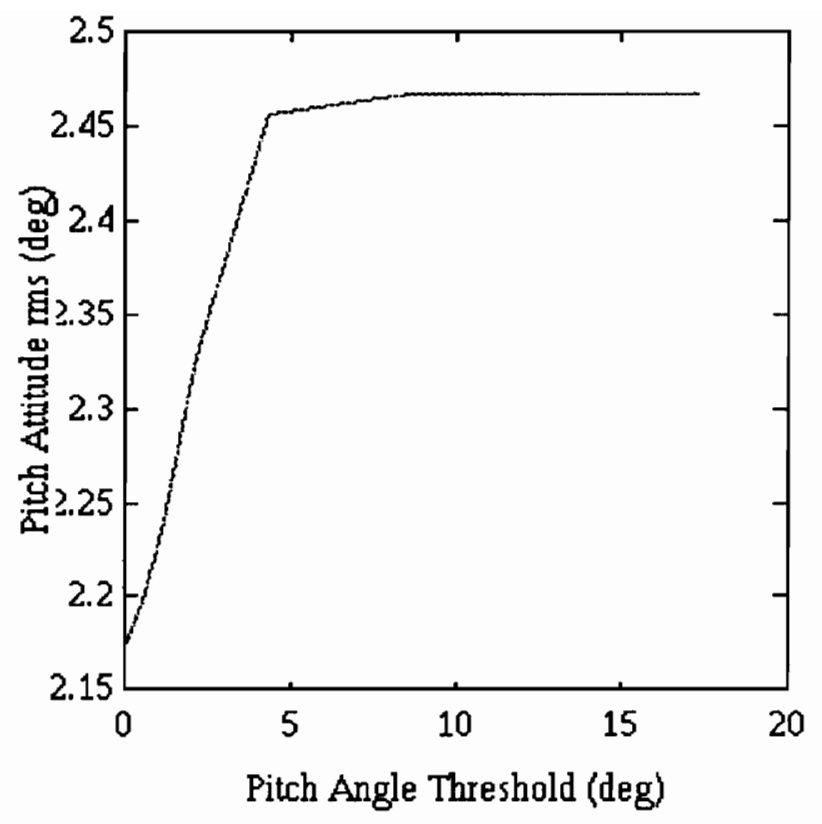

Figure 26-a: Pitch Attitude rms vs. Attitude Threshold

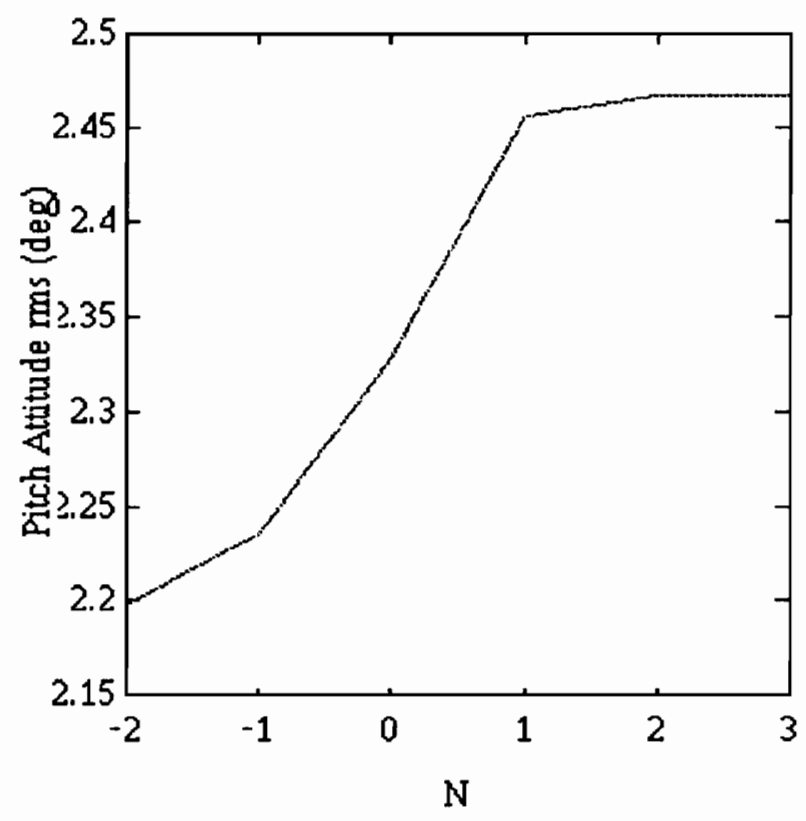

Figure 26-b: Pitch Attitude rms vs. N 


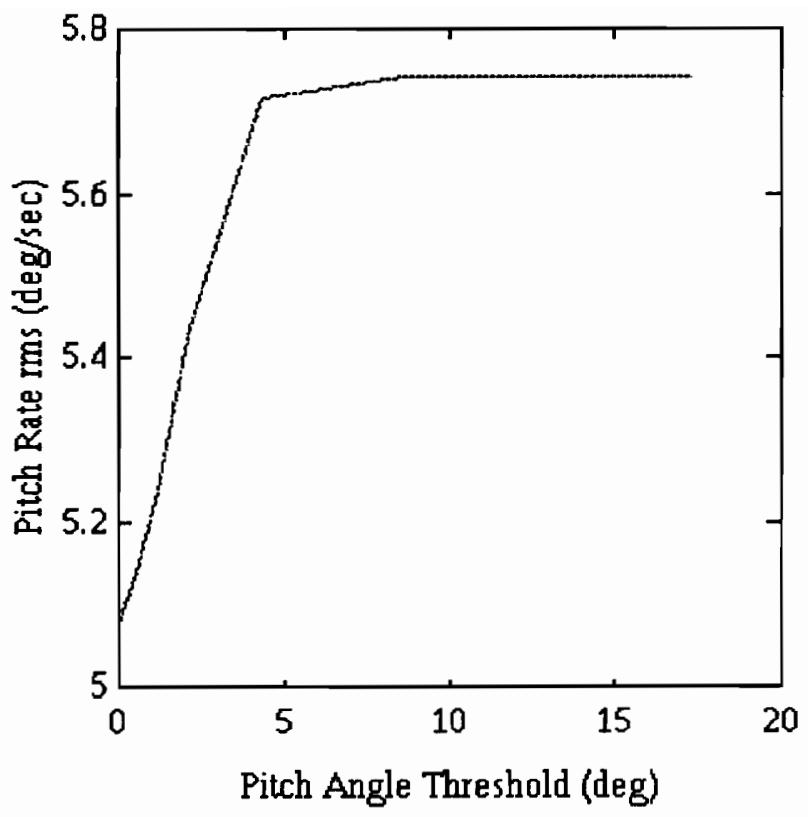

Figure 27-a: Pitch Rate rms vs. Attitude Threshold

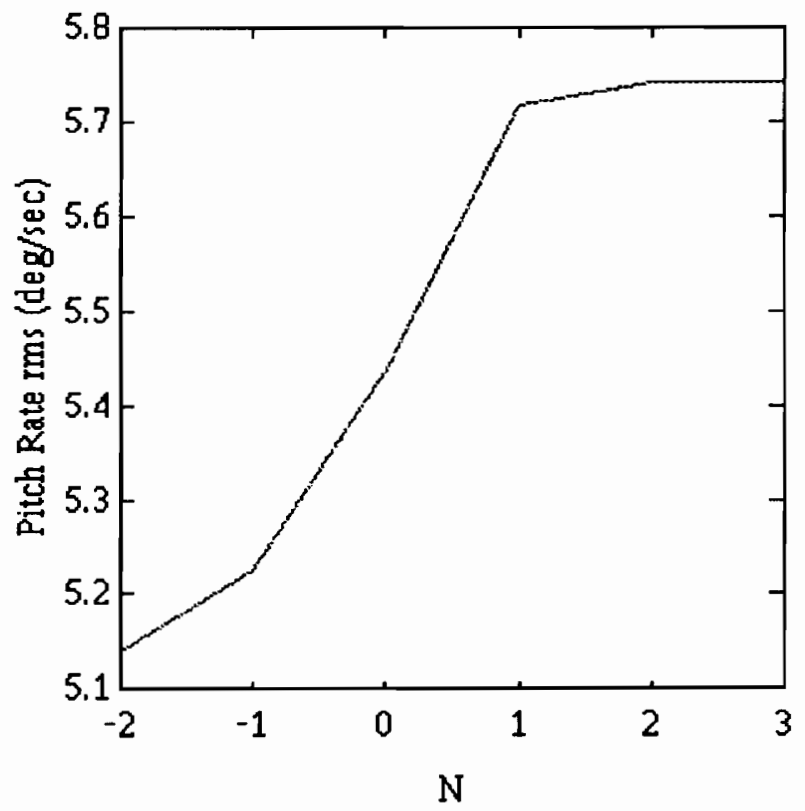

Figure 27-b: Pitch Rate rms vs. N 


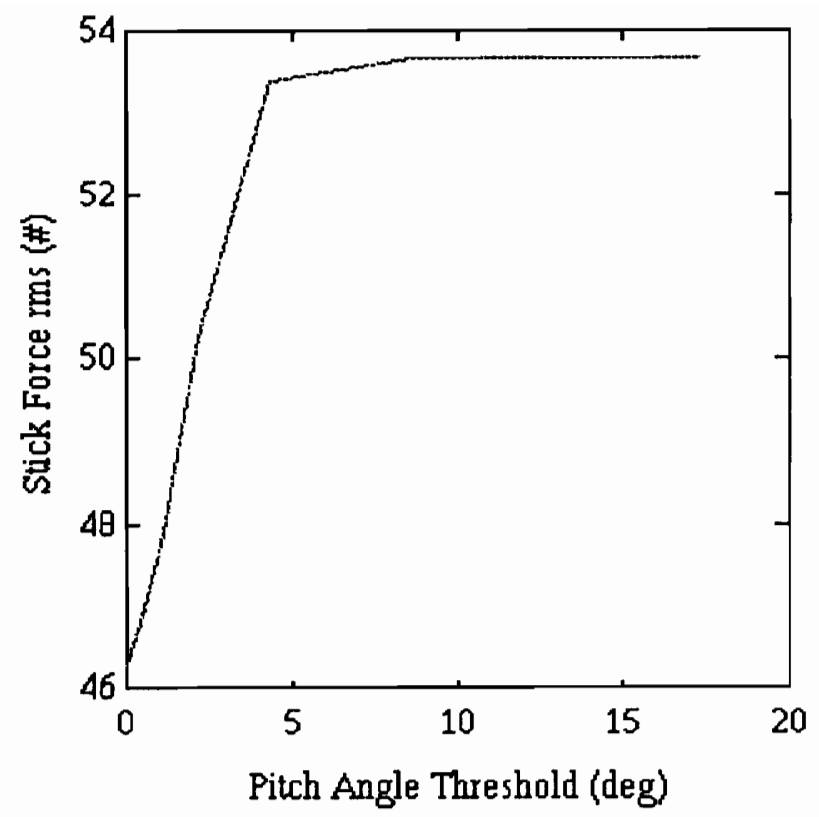

Figure 28-a: Stick Force vs. Attitude Threshold

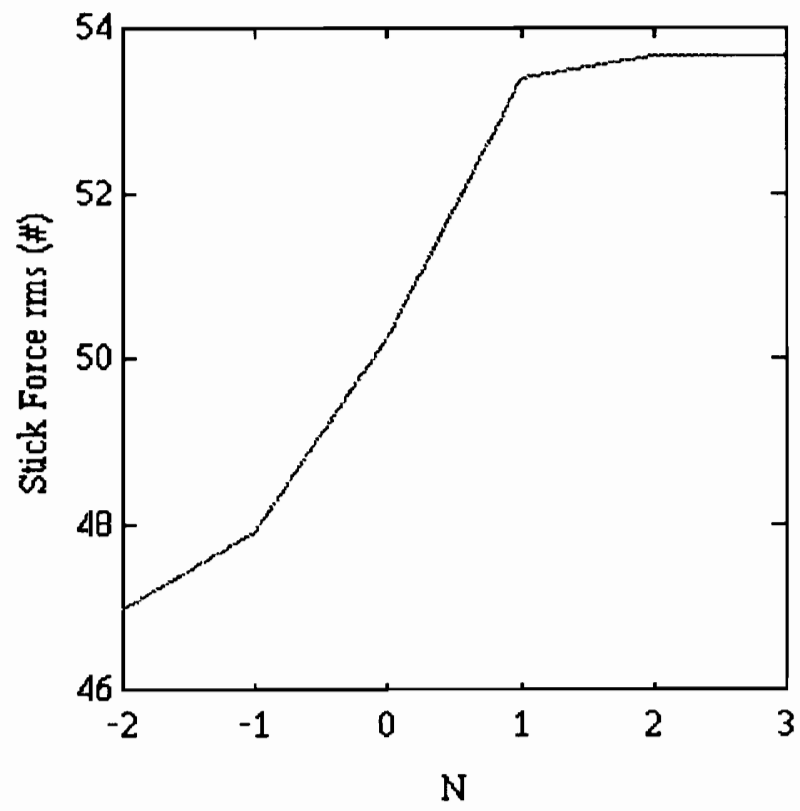

Figure 28-b: Stick Force vs. N 


\subsection{Vita}

The author was born on December 24, 1970 in Chicago, Illinois. He graduated from Tazewell High School in June, 1988 and then entered Virginia Polytechnic Institute and State University in August, 1988. He received a Bachelor of Science degree in Aerospace Engineering in May, 1992. In August, 1992, he enrolled for graduate study in Aerospace Engineering at Virginia Polytechnic Institute and state University and received a Master of science degree in December, 1993.

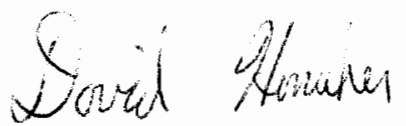

